\title{
NEW EXTREMAL PROBLEMS FOR THE RIEMANNIAN RECOGNITION PROGRAM VIA ALEXANDROV GEOMETRY
}

\author{
KARSTEN GROVE AND STEEN MARKVORSEN
}

In its most general form, the recognition problem in riemannian geometry asks for the identification of an unknown riemannian manifold via measurements of metric invariants on the manifold. Optimally one wants to recognize a manifold having made as few measurements as possible. Many results in riemannian geometry, including pinching theorems, can be viewed this way. Here we are only interested in measurements that assign real numbers to each (complete) riemannian manifold. Typical examples of such invariants are $d i$ ameter, volume, curvature bounds, etc. When viewing one or several invariants, $I=\left(I_{1}, \cdots, I_{l}\right)$, of this type as a map on a suitable class, $\mathscr{M}$, of riemannian manifolds, the following problems pose themselves:

(1) The range problem, i.e., what is $I(\mathscr{M}) \subset \mathbb{R}^{l}$ ?

(2) Slicing problems, i.e., what can be said about $I^{-1}(\Omega), \Omega \subset \mathbb{R}^{l}$ ?

(3) Extremal problems, i.e., recognize or describe the structure of manifolds $M$ in $\mathscr{M}$ with $I(M)$ close to the boundary of $I(\mathscr{M})$ when nonempty.

In order to attack extremal problems, one first needs to solve the range problem. This is not the case for slicing problems, where typical answers are provided by finiteness theorems. These, on the other hand, can be interpreted as a first step in the full recognition process. To proceed in this process it is necessary to make more measurements, i.e., to start the program over again for the smaller class consisting only of finitely many "types" (cf. also the discussion in [G]).

In this paper we point out several interesting invariants and initiate the study of their importance for the recognition program by establishing a number of specific range and extremality properties.

For $q \geq 2$, we define the $q^{\text {th }}$ packing radius, pack $k_{q}$, of a compact metric space $X$ as the largest $r>0$ for which $X$ contains $q$ disjoint open $r$-balls, i.e.,

$$
\operatorname{pack}_{q} X=\frac{1}{2} \max _{x_{1} \cdots x_{q}} \min _{1 \leq i<j \leq q} \operatorname{dist}\left(x_{i}, x_{j}\right),
$$

Received by the editors December 2, 1992 and, in revised form, July 12, 1993.

1991 Mathematics Subject Classification. Primary 51Kxx, 53C20, 53C45, 53C23.

This work was supported in part by grants from the National Science Foundation and the Danish Natural Sciences Research Council. 
where the maximum is taken over all configurations of $q$ points in $X$. Clearly

$$
\frac{1}{2} \operatorname{diam} X=\operatorname{pack}_{2} X \geq \cdots \geq \operatorname{pack}_{q} X \geq \cdots
$$

and $\lim _{q \rightarrow \infty}$ pack $X=0$. Any configuration of points in $X$ which realizes pack $_{q} X$ is called a q-packer.

For each $q \geq 2$, we define the q-extent, $x t_{q} X$, of a compact metric space $X$ to be the maximal average distance between the points in $q$-tuples of $X$, i.e.,

$$
x t_{q} X=\max _{x_{1} \cdots x_{q}} x t_{q}\left(x_{1}, \cdots, x_{q}\right),
$$

where $x t_{q}: X^{q} \rightarrow \mathbb{R}$ is the q-extent function

$$
x t_{q}\left(x_{1}, \cdots, x_{q}\right)=\left(\begin{array}{l}
q \\
2
\end{array}\right)^{-1} \sum_{i<j} \operatorname{dist}\left(x_{i}, x_{j}\right)
$$

for any $q$-tuple $\left(x_{1}, \cdots, x_{q}\right) \in X^{q}$. Now clearly

$$
\operatorname{diam} X=x t_{2} X \geq x t_{3} X \geq \cdots \geq x t_{q} X \geq \cdots
$$

and we refer to the limit

$$
x t X=\lim _{q \rightarrow \infty} x t_{q} X
$$

as the extent of $X$. Any configuration of $q$ points in $X$ which realizes $x t_{q} X$ is called a q-extender.

The extents are instrumental in estimating the number of isolated fixed points for isometric group actions in the presence of a lower curvature bound. If, for example, $G$ acts on a positively curved $n$-manifold $M$ with $(q+1)$ isolated fixed points $p_{0}, \cdots, p_{q}$, then an angle counting and comparison argument in $M / G$ yields

$$
\frac{1}{q+1} \sum_{i=0}^{q} x t_{q}\left(\frac{S_{p_{i}}^{n-1}}{G}\right)>\frac{\pi}{3} .
$$

This shows in particular that any isometric circle action on a positively curved 4-manifold can have at most three isolated fixed points (see [HK]).

Our aim here, however, is to investigate the role of the invariants $x t_{q}$ and pack $_{q}$ in the recognition program. In particular, by (2) and (5) they give rise to natural filtrations of any class $\mathscr{X}$ of metric spaces, $\mathscr{Z} \supset F_{d}^{2}(\mathscr{X}) \supset \cdots \supset$ $F_{d}^{q}(\mathscr{X}) \supset \cdots$, where, e.g., $F_{d}^{q}(\mathscr{X})=\left\{X \in \mathscr{X} \mid x t_{q} X \geq d\right\}$. If, for example, $\mathscr{M}_{1}$ is the class of closed riemannian manifolds $M$ with sectional curvature, $\sec M \geq 1$ and $d=\frac{\pi}{2}$, the work in [GG] can be used to describe this filtration explicitly, and gives among other things a characterization of projective spaces.

When $\mathscr{Z}$ is the class of all length spaces (metric spaces in which distances are measured by the infimum of lengths of curves), the range problem for $x t_{q}$ as well as for pack $_{q}$ has a trivial and therefore not so interesting solution (cf. 
Examples 1.6). This remains true even for the subclass $\mathscr{M}$ of all closed riemannian manifolds. Here, however, having maximal q-extent (or q-packing radius), i.e., $x t_{q} M=\operatorname{diam} M\left(2 \operatorname{pack}_{q} M=\operatorname{diam} M\right)$ for large $q$, is only possible if minsec $M$ is very negative or if $\operatorname{dim} M$ is large. For these reasons we focus our attention on the class $\mathscr{M}_{k}(n)$ of closed riemannian $n$-manifolds $M$ with $\sec M \geq k$ and more generally on the class $\mathscr{A}_{k}(n)$ of all compact Alexandrov spaces $X$ with $\operatorname{dim} X \leq n$ and $\operatorname{curv} X \geq k$. (We use the terminology Alexandrov space for any complete finite Hausdorff dimensional length space with curvature bounded below in local distance comparison sense.) The importance of such spaces is in part based on the fact that any space $Y$ in the Gromov-Hausdorff closure of $\mathscr{M}_{k}(n)$ is an Alexandrov space with $\operatorname{dim} Y \leq n$ and curv $Y \geq k$ (cf. [GP3]). This combined with convergence techniques initiated in [G1] and developed in [Pt1], [GPW], [Y], and most recently in [P] allows one to attack extremal problems in $\mathscr{M}_{k}(n)$ by first solving corresponding extremal/rigidity problems in $\mathscr{A}_{k}(n)$.

The most important new technical tool used in the proofs of our main results is a rigidity version of Toponogov's distance comparison theorem for Alexandrov spaces (cf. Theorem 2.4 and the Appendix).

The following analogue of Toponogov's maximal diameter theorem provides a solution to the range and extremal problems for each individual $x t_{q}$ on $\mathscr{A}_{1}$ :

Theorem A. Let $X$ be an $n$-dimensional, $n \geq 2$, Alexandrov space with curv $X$ $\geq 1$. Then

$$
x t_{q} X \leq x t_{q} S_{1}^{n}
$$

for all $q \geq 2$. Moreover, equality holds for some $q$ if and only if diamX $=\pi$, i.e., $X$ is isometric to the spherical suspension $\Sigma_{1} E$ of an $(n-1)$-dimensional Alexandrov space $E$ with curv $E \geq 1$.

We also point out the remarkable fact that in contrast to $\operatorname{pack}_{q} S_{1}^{n}$ (cf. [GW]), $x t_{q} S_{1}^{n}$ is independent of $n$ (cf. Theorem 1.8).

When $x t_{q}$ is restricted to $\mathscr{A}_{1}$ as in Theorem A above, it is easy to see that it cannot be maximal, i.e., $x t_{q}=\operatorname{diam} X$, unless $\operatorname{diam} X \leq \frac{\pi}{2}$. In the extreme case where $\operatorname{diam} X=\frac{\pi}{2}$ and $q=n+1$, we have as our first main result the following rigidity theorem (cf. Theorem 2.13).

Theorem B. Let $X$ be an n-dimensional Alexandrov space with curv $X \geq 1$. Then $x t_{n+1} X=\operatorname{diam} X=\frac{\pi}{2}$ if and only if $X$ is isometric to $S_{1}^{n} / H$, where $H$ is a finite abelian group of isometric involutions in $O(n+1)$ acting without fixed points on $S_{1}^{n}$.

It turns out that only a few spaces among $X=S_{1}^{n} / H$ are (generalized) manifolds. In fact such an $X$ is either isometric to $R P_{1}^{n}$ or homeomorphic to $S^{n}$ (cf. Theorem 2.14 and Remark 2.15). This yields the following generalization of the main theorems in [GP2] and [OSY]:

Corollary C. Fix an integer $n \geq 2$. There is an $\varepsilon=\varepsilon(n)>0$ such that any closed riemannian n-manifold $M$ with sec $M \geq 1$ and $x t_{n+1} M \geq \frac{\pi}{2}-\varepsilon$ is homeomorphic to $S^{n}$ or diffeomorphic to $R P^{n}$. If $x t_{n+1} M=\operatorname{diam} M=\frac{\pi}{2}$, then $M$ is isometric to $R P_{1}^{n}=S_{1}^{n} / \mathbb{Z}_{2}$. 
Since $M$ is homeomorphic to $S^{n}$ if $\sec M \geq 1$ and $\operatorname{diam} M>\frac{\pi}{2}$, we can assume that $\operatorname{diam} M \leq \frac{\pi}{2}$ in the above theorem. For such manifolds we know from the discussion above that $M$ is in fact Gromov-Hausdorff close to either $R P_{1}^{n}$ or to some singular space homeomorphic to $S^{n}$ provided $x t_{n+1} M \geq \frac{\pi}{2}-\varepsilon$. The conclusion then follows from [Y], [GP1], [H], and the generalized Poincare conjecture (cf. also [GPW] or [P]). We also point out that Theorem B and Corollary $\mathrm{C}$ hold as well with $x t_{n+1}$ replaced by 2 pack $_{n+1}$.

Yet another type of interesting extremal problem is obtained by relaxing the lower curvature bound to the general bound curv $X \geq k$. In this generality we cannot use $k$ as a normalizing invariant as in the previous theorems. Instead we normalize all spaces to have fixed radius, $\operatorname{rad} X=\pi$, where by definition $\operatorname{rad} X=\min _{p} \max _{q} \operatorname{dist}(p, q)$, and consequently $k \leq 1$.

Now, if we let $D_{k}^{n}(\pi)$ denote the closed disk of radius $\pi$ in the simply connected $n$-dimensional space form, $S_{k}^{n}$ of constant curvature $k \leq 1$, then distance comparison arguments yield

$$
x t_{q} X \leq x t_{q} D_{k}^{n}(\pi), \quad q \geq 2,
$$

and

$$
\operatorname{pack}_{q} X \leq \operatorname{pack}_{q} D_{k}^{n}(\pi), \quad q \geq 2,
$$

for every compact $n$-dimensional Alexandrov space with curv $X \geq k$ and $\operatorname{rad} X$ $\leq \pi$ (cf. (3.1)). Although these inequalities are trivially optimal for Alexandrov spaces, they are not optimal for riemannian manifolds.

For $k<\frac{1}{4}$, let $\Delta_{k}^{n}$ denote the maximal regular $n$-simplex inscribed in $D_{k}^{n}(\pi)$. Note that for each $n \geq 2$, the inradius $r_{n}(k)$ of $\Delta_{k}^{n}$ defines a strictly increasing continuous function $r_{n}:\left(-\infty, \frac{1}{4}\right) \rightarrow(0, \pi)$. In particular, there is exactly one value $k_{n} \in\left(-\infty, \frac{1}{4}\right)$ such that $r_{n}\left(k_{n}\right)=\frac{\pi}{2}$. A calculation gives

$$
0=k_{2}<k_{3}<\cdots<k_{n}=\left(\frac{2 \arctan \sqrt{1-2 / n}}{\pi}\right)^{2}<\cdots<\lim _{n \rightarrow \infty} k_{n}=\frac{1}{4} .
$$

We are now ready to state our second main result, which in the riemannian category addresses all three problems of the recognition program for the $(n+1)$ packing radius.

Theorem D. Let $X$ be an $n$-dimensional Alexandrov space with curv $X \geq k$, radX $=\pi$, and pack ${ }_{n+1} X=$ pack $_{n+1} D_{k}^{n}(\pi)$. If in addition $X$ is a closed manifold (or Poincaré duality space), then $k=1$ or $k \leq k_{n}$. Moreover, if $k=1$, then $X$ is isometric to $S_{1}^{n}$, and if $k=k_{n}$ it is isometric to the double $D \Delta_{k_{n}}^{n}=\Delta_{k_{n}}^{n} \cup \Delta_{k_{n}}^{n} /\left(\partial \Delta_{k_{n}}^{n} \sim \partial \Delta_{k_{n}}^{n}\right)$ of $\Delta_{k_{n}}^{n}$.

This result combined with the stability theorems in [GPW], $n \neq 3$, or [P] gives the following corollary for riemannian manifolds.

Corollary E. For any integer $n \geq 2$ and real number $k \leq 1$, let $\mathscr{M}_{k}(n, \pi)$ be the class of closed riemannian n-manifolds $M$ satisfying secM $\geq k$ and $\operatorname{rad} M=\pi$. Then 
(1) For every $\varepsilon>0$ there are at most finitely many homeomorphism types among $M \in \mathscr{M}_{k}(n, \pi)$ with

$$
\operatorname{pack}_{n+1} M \geq \operatorname{pack}_{n+1} D_{k}^{n-1}(\pi)+\varepsilon .
$$

(2) The inequality

$$
\operatorname{pack}_{n+1} M \leq \operatorname{pack}_{n+1} D_{k}^{n}(\pi), \quad M \in \mathscr{M}_{k}(n, \pi)
$$

is optimal if and only if $k \in\left(-\infty, k_{n}\right] \cup\{1\}$.

(3) There is an $\varepsilon=\varepsilon(n)$, such that any $M \in \mathscr{M}_{k_{n}}(n, \pi)$ for which

$$
\operatorname{pack}_{n+1} M \geq \operatorname{pack}_{n+1} D_{k_{n}}^{n}(\pi)-\varepsilon
$$

is homeomorphic to $S^{n}$.

Our main results deal mostly with extremal problems for $q$-extents, or $q$ packing radii, when $q \leq \operatorname{dim} M+1$. The possible distribution of points that realize the $q$-extents for $q$ large seems to be a fascinating problem, supported in part by examples. In spaces that are fairly symmetric it appears that there are many such solutions as $q \rightarrow \infty$ (cf. $S_{1}^{n}$ in $\S 1$ ). Indeed, it seems that these distributions, i.e., the extenders, will pick up asymmetries of a space. Thus, although by definition the $q$-extents of a space like its $q$-packing radii might be viewed upon as size invariants, they should probably be thought of as global shape invariants, much in the same way that curvatures are thought of as local shape invariants. This is supported also by the surprising fact (1.12) that the limit of $q$-extents as $q \rightarrow \infty$ is related to the excess as defined in [GP4] (cf. also $[\mathrm{AG}]$ ). However, we leave it for the future to see the possible germination of these ideas.

The paper is divided into three sections and one appendix:

$\S 1$. The family of $q$-extents and examples.

$\S 2$. Positively curved spaces with large extents.

$\S 3$. Alexandrov manifolds with bounded radius and large packing radii.

Appendix. Rigidity from distance comparison in Alexandrov spaces.

The above results were announced in [GM] except that Theorem D and its corollary were stated incorrectly with $x t_{n+1}$ instead of $p a c k_{n+1}$. The error was caused by an incorrect computation of the $k_{n}$ 's in (9). The packing radius results in $[\mathrm{GM}]$ will appear as part of [GW].

It is our pleasure to thank S. Ferry for suggestions that led to the proof of Lemma 3.5 given here, and I. Madsen for explaining the topological significance of Grothendieck groups of positively curved spaces (cf. 2.16 and 2.17). Finally, we thank G. Perelman and the referee for constructive criticism of the first version of the manuscript, which prompted a significant expansion of $\S 2$ and an overall improvement of the exposition.

\section{The Family of $q$-eXTents AND EXamples}

Before computing $x t_{q} X$ in a few examples, we derive some useful inequalities. First, by repeating each point of a $q$-extender $p$ times we get

$$
x t_{p q} X \geq \frac{p^{2} \cdot x t_{q} X \cdot\left(\begin{array}{l}
q \\
2
\end{array}\right)}{\left(\begin{array}{c}
p q \\
2
\end{array}\right)}=\frac{p(q-1)}{p q-1} x t_{q} X .
$$


As immediate consequences we have

$$
\begin{gathered}
x t_{2 p} X \geq \frac{p}{2 p-1} \operatorname{diam} X, \\
x t X=\lim _{p} x t_{p q} X \geq \frac{q-1}{q} x t_{q} X,
\end{gathered}
$$

and in particular

$$
\frac{1}{2} \operatorname{diam} X \leq x t X<\operatorname{diam} X
$$

In analogy to (1.2) we have

$$
x t_{2 p+1} X \geq \frac{p+1}{2 p+1} \operatorname{diam} X .
$$

The following examples show that all of these inequalities are optimal.

Examples 1.6. (i) Let $X=S^{1}$ be the unit circle in $\mathbb{R}^{2}$ with its usual riemannian distance. Suppose $\left(x_{1}, \cdots, x_{q}\right)$ is a $q$-extender in $S^{1}$. If $\left(x_{1}, \cdots, x_{q}\right)$ contains a pair of antipodal points, an easy calculation shows that the remaining set then necessarily is a $(q-2)$-extender. Moreover, this extender together with any other pair of antipodal points is also a $q$-extender for $S^{1}$. Therefore, assume $\left(y_{1}, \cdots, y_{n}\right)$ is an $n$-extender in $S^{1}$ which does not contain any pair of antipodal points. By a straightforward variation argument the line through any $y_{i}$ and the origin separates $\left(y_{1}, \cdots, y_{n}\right)-y_{i}$ into sets of equal cardinality. Furthermore, the set obtained by moving only one of the $y_{i}$ 's in its arc determined by the antipodal image of $\left(y_{1}, \cdots, y_{n}\right)$ remains a $q$-extender (see Figure 1.7).

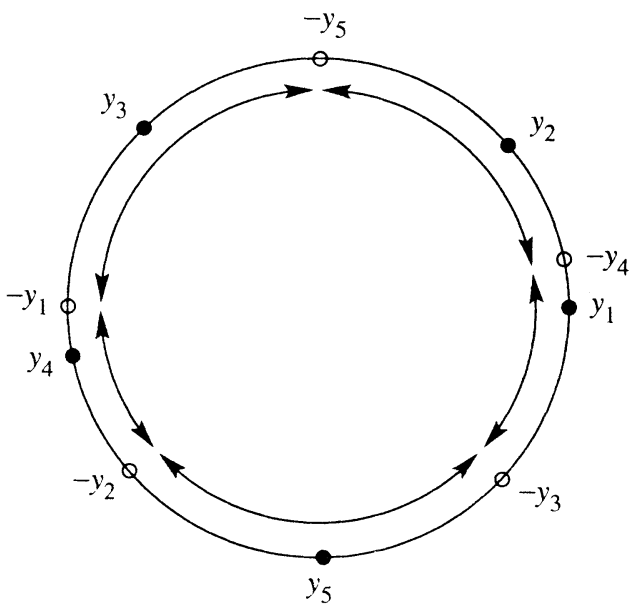

FIGURE 1.7 
We conclude that any $2 p$-extender consists of pairs of antipodal points. Thus

$$
x t_{2 p}\left(S^{1}\right)=\frac{p}{2 p-1} \pi \text {. }
$$

Similarly, any $(2 p+1)$-extender consists of pairs of antipodal points together with a configuration of points satisfying the separation condition alluded to above. Thus

$$
x t_{2 p+1}\left(S^{1}\right)=\frac{p+1}{2 p+1} \pi
$$

and in particular

$$
x t\left(S^{1}\right)=\frac{\pi}{2}=\frac{1}{2} \operatorname{diam} S^{1} .
$$

(ii) Let $X=[0, \pi]$ with $\operatorname{dist}\left(\theta_{1}, \theta_{2}\right)=\left|\theta_{1}-\theta_{2}\right|$. It follows immediately from the $S^{1}$-discussion above that

$$
x t_{q}[0, \pi]=x t_{q} S^{1}, \text { all } q .
$$

In addition the possible extenders consist of an equal number of points at the endpoints of $[0, \pi]$ and possibly one point anywhere on $[0, \pi]$.

(iii) Let $X=V_{k}(\theta)$ be the one point union of $k$ intervals $[0, \theta]$ identified at 0 , and equipped with the induced length metric. Arguing as in (i) and (ii) above we conclude that any $q$-extender, $q=p k+n, 0 \leq n<k$, for $V_{k}(\theta)$ has its points evenly distributed among the $k$ endpoints of $V_{k}(\theta)$. Therefore

$$
x t_{q} V_{k}(\theta)=\frac{q(q-p)-n(p+1)}{q(q-1)} \operatorname{diam} V_{k} \text {. }
$$

In particular $V_{k}$ has maximal $k$-extent, and

$$
x t V_{k}=\frac{k-1}{k} \text { diam } V_{k} .
$$

(iv) Let $X=R P_{1}^{n}$ be the real projective $n$-space with metric of constant curvature 1 . Obviously $x t_{n+1} R P^{n}=\frac{\pi}{2}=\operatorname{diam} R P^{n}$, i.e., $R P^{n}$ has maximal $(n+1)$-extent. Thus $x t R P^{n} \geq \frac{n}{n+1} \operatorname{diam} R P^{n}$.

Note that (i) and (ii) above provide examples where all extents are minimal, whereas (iii) and (iv) are examples exhibiting maximal extents.

Our description of the $q$-extenders of $S^{1}$ in Example 1.6 (i) can be lifted to the higher dimensional unit spheres $S_{1}^{n}, n \geq 2$. For $n=2$ the result below was obtained previously by $F$. Nielsen in $[N]$. We shall follow the same method of proof and make use of a fundamental observation due to I. Fary [F].

Theorem 1.8. For all integers $n \geq 1$ and $q \geq 2$ we have:

(i) $x t_{q}\left(S_{1}^{n}\right)=x t_{q}\left(S_{1}^{1}\right)=\pi /\left(2-\left\lfloor\frac{q+1}{2}\right\rfloor^{-1}\right)$.

(ii) Those points of a q-extender which do not appear in antipodal pairs will all lie on a great circle in such a way that they realize an $S^{1}$-extender on that circle. In particular, if $q$ is even, every q-extender consists of pairs of antipodal points. 
Proof. For $1 \leq l \leq n$ and $q \geq 2$, let $Q(l)$ be a collection of $q$ points in $S_{1}^{l} \subset \mathbb{R}^{l+1}$ (some of the points in $Q(l)$ may coincide, in which case we count multiplicities). We denote by $\hat{Q}(l)$ the union of $Q(l)$ and its antipodal set, $-Q(l)$ in $S_{1}^{l}$, and set $\hat{S}_{1}^{l}=S_{1}^{l}-\hat{Q}(l)$. Now, for each $x \in \hat{S}_{1}^{l}$ define

$$
\varphi_{x}: S^{l}-\{x,-x\} \rightarrow S_{1}^{l-1}
$$

to be the map $\varphi_{x}=r_{x} \circ \pi_{x}$, where $\pi_{x}$ is the restriction to $S_{1}^{l}$ of the orthogonal projection in $\mathbb{R}^{l+1}$ onto the tangent space $T_{x} S_{1}^{l}=\mathbb{R}^{l}$, and $r_{x}: T_{x} S_{1}^{l}-\{0\} \rightarrow$ $S_{1}^{l-1}$ is the radial projection of $T_{x} S_{1}^{l}-\{0\}$ onto the unit sphere in $T_{x} S_{1}^{l}$.

A result of Fary enables us to compare the average distance of $Q(l)$ in $S_{1}^{l}$, i.e., $x t_{q} Q(l)$, with the corresponding average distances $x t_{q} \varphi_{x}(Q(l)), x \in \hat{S}_{1}^{l}$, in $S_{1}^{l-1}$. Precisely, if for any unit sphere we use $d$ to denote the spherical distance, then following $[\mathrm{F}]$ we have for any $\zeta_{1}, \zeta_{2} \in Q(l)$

$$
d\left(\zeta_{1}, \zeta_{2}\right)=\frac{1}{\omega_{l}} \int_{\hat{S}_{1}^{l}} d\left(\varphi_{x}\left(\zeta_{1}\right), \varphi_{x}\left(\zeta_{2}\right)\right) d x
$$

where $\omega_{l}=$ vol $S_{1}^{l}$. Indeed, replacing $\zeta_{1}, \zeta_{2}$ by any $p_{1}, p_{2} \in S_{1}^{l}$, we claim that the same formula holds when the integral is taken over $\hat{S}_{1}^{l}-\left\{\hat{p}_{1}, \hat{p}_{2}\right\}$. To see this observe that the integral is a function $f$ of $\theta=d\left(p_{1}, p_{2}\right) \in[0, \pi]$ only, and that $f(0)=0, f(\pi)=\pi$, and $f(\theta)=f\left(\theta_{1}\right)+f\left(\theta_{2}\right)$ whenever $\theta=$ $\theta_{1}+\theta_{2}, \theta_{i} \in[0, \pi]$ (pick $p_{0} \in S_{1}^{l}$ so that $\theta_{1}=d\left(p_{1}, p_{0}\right)$ and $\theta_{2}=d\left(p_{0}, p_{2}\right)$ ). By continuity, $f(\theta)=\theta$ for all $\theta \in[0, \pi]$, and in particular (1.10) follows.

Now starting with a collection $Q(n)$ of $q$ points in $S_{1}^{n}$, we can express individual distances by iterated applications of (1.10): Let $T_{n-2}=T_{1}^{v} \cdots T_{1}^{v} T_{1} S_{1}^{n}$ be the $(n-2)$-iterated unit vertical tangent-bundle of $S_{1}^{n}$, i.e., as a bundle, $T_{n-2}$ is a 2 -sphere bundle over a 3 -sphere bundle over ... an $(n-1)$-sphere bundle over $S_{1}^{n}$, and measure theoretically,

$$
T_{n-2}=S_{1}^{n} \times S_{1}^{n-1} \times \cdots \times S_{1}^{2} .
$$

As before, to $\hat{Q}(n)$ there corresponds a measure zero subset $\hat{Q}_{n-2} \subset T_{n-2}$, and

$$
\hat{T}_{n-2}=T_{n-2}-\hat{Q}(n)_{n-2} \quad\left(\hat{Q}(n)_{1}=\left.\left(\bigcup_{x \in \hat{S}_{1}^{n}} \varphi_{x}(\hat{Q}(n))\right) \cup T_{1} S_{1}^{n}\right|_{\hat{Q}(n)} \text { etc. }\right) .
$$

Also for each $\underline{\mathrm{x}} \in \hat{T}_{n-2}$ we get a map $\varphi_{\underline{\underline{x}}}: \hat{S}_{1}^{n} \rightarrow S_{1}^{1}$ by repeating the construction from (1.9). Thus, (1.10) yields

$$
d\left(\zeta_{i}, \zeta_{j}\right)=\frac{1}{V o l T_{n-2}} \int_{\hat{T}_{n-2}} d\left(\varphi_{\underline{\mathbf{x}}}\left(\zeta_{i}\right), \varphi_{\underline{\mathbf{x}}}\left(\zeta_{j}\right)\right) d \underline{\mathbf{x}}
$$

for any $\zeta_{i}, \zeta_{j} \in Q(n)$. 
In particular, if $Q(n)$ is a $q$-extender for $S_{1}^{n}$, then

$$
\begin{aligned}
x t_{q}\left(S_{1}^{n}\right) & =\left(\begin{array}{l}
q \\
2
\end{array}\right)^{-1} \sum_{i<j} d\left(\zeta_{i}, \zeta_{j}\right) \\
& =\left(\left(\begin{array}{l}
q \\
2
\end{array}\right) \operatorname{vol}\left(T_{n-2}\right)\right)^{-1} \int_{\hat{T}_{n-2}} \sum_{i<j} d\left(\varphi_{\underline{\mathbf{x}}}\left(\zeta_{i}\right), \varphi_{\underline{\mathbf{x}}}\left(\zeta_{j}\right)\right) d \underline{\mathbf{x}} \\
& \leq\left(\left(\begin{array}{l}
q \\
2
\end{array}\right) \operatorname{vol}\left(T_{n-2}\right)\right)^{-1} \int_{\hat{T}_{n-2}}\left(\begin{array}{l}
q \\
2
\end{array}\right) x t_{q}\left(S_{1}^{1}\right) d \underline{\mathbf{x}} \\
& =x t_{q}\left(S_{1}^{1}\right) .
\end{aligned}
$$

Since, however, obviously $x t_{q}\left(S_{1}^{n}\right) \geq x t_{q}\left(S_{1}^{1}\right)$, we conclude that $x t_{q}\left(S_{1}^{n}\right)=$ $x t_{q}\left(S_{1}^{1}\right)$ and the above inequality is an equality.

The discussion above also allows us to characterize $q$-extenders $Q(n)$ in $S_{1}^{n}$. First observe that if for $\underline{\mathrm{x}} \in \hat{T}_{n-2}$ we let $\underline{\mathrm{x}}_{0}, \underline{\mathrm{x}}_{1}, \cdots, \underline{\mathrm{x}}_{n-2}=\underline{\mathrm{x}}, \underline{\mathrm{x}}_{n-i} \in \hat{T}_{n-i}$, denote the corresponding iterated projections of $\mathrm{x} \in \hat{T}_{n-2} \rightarrow \hat{T}_{n-3} \rightarrow \cdots \rightarrow$ $\hat{T}_{1} S_{1}^{n} \rightarrow \hat{T}_{0}=\hat{S}_{1}^{n}$, then by the equality above, $\varphi_{\underline{x}_{n-i}}(Q(n))=Q(i-1) \subset \hat{S}_{1}^{i-1}$ is a $q$-extender in $S_{1}^{i-1}$ for all $\underline{\mathrm{x}} \in \hat{T}_{n-2}$ and all $i=2, \cdots, n$.

Assume by induction that the statement in (ii) holds in all dimensions $2, \cdots$, $n-1$ and let $Q(n)$ be a $q$-extender in $S_{1}^{n}$. Pick $\underline{x} \in \hat{T}_{n-2}$ and consider the corresponding $q$-extender $\varphi_{\underline{x}_{0}}(Q(n))=Q(n-1) \subset S_{1}^{n-1}$. If $\Lambda(n-1) \subset Q(n-1)$ is the subset where all antipodal pairs have been removed, then $\Lambda(n-1)$ is contained in a great circle $S_{1}^{1} \subset S_{1}^{n}$. By definition of $\varphi_{\underline{\mathbf{x}}_{0}}: \hat{S}_{1}^{n} \rightarrow S_{1}^{n-1}$ it follows that $\Lambda(n) \subset S_{1}^{2} \subset S_{1}^{n-1}$ and hence $\Lambda(n) \subset S_{1}^{1}$ by [N].

Since we have all the necessary ingredients, and Nielsen's proof was presented in Danish, we give it here for completeness. As above consider a $\lambda$-extender $\Lambda(2) \subset S_{1}^{2}$ which does not contain any pair of antipodal points. It follows from the separation property of the $\lambda$-extenders $\varphi_{x}(\Lambda(2)) \subset S_{1}^{1}$ (cf. Example 1.6 (i)) that every 2-plane in $\mathbb{R}^{3} \supset S_{1}^{2}$ through the center of $S_{1}^{2}$ and one of the points in $\Lambda(2)$ must separate the rest of $\Lambda(2)$ into sets of equal cardinality. In effect, any such plane which contains two points from $\Lambda(2)$ must at least contain one more (third) point from $\Lambda(2)$, and hence all of $\Lambda(2)$ is in fact contained in one 2-plane by the Sylvester-Gallai theorem [C, p.65].

In contrast to our discussion of sphere-extenders, our present understanding of disk-extenders in general is far from complete. For example we know that ext ${ }_{n+1} D_{k}^{n}(\pi)$ is realized by the vertices of a maximal regular inscribed $n$-simplex $\Delta_{k}^{n}$ in $D_{k}^{n}(\pi)$ only when $k \leq \frac{1}{16}$, and that this cannot be true for $k$ arbitrarily close to $\frac{1}{4}$ by Theorem 1.8 .

Our main concern in $\S 2$ will be spaces with large extents. First, however, we discuss the other extreme, i.e., what is the significance of having small extents? For this purpose fix two points $x_{1}, x_{2} \in X$ with $\operatorname{dist}\left(x_{1}, x_{2}\right)=\operatorname{diam} X$. Let 
$x_{0} \in X$ be a maximum point for the excess function (cf. [AG]),

$$
e_{x_{1} x_{2}}(x)=\operatorname{dist}\left(x_{1}, x\right)+\operatorname{dist}\left(x, x_{2}\right)-\operatorname{dist}\left(x_{1}, x_{2}\right),
$$

and set $\alpha=\operatorname{dist}\left(x_{1}, x_{0}\right), \beta=\operatorname{dist}\left(x_{0}, x_{2}\right)$. By placing $p$ points at $x_{1}, p$ points at $x_{2}$, and one point at $x_{0}$, we derive

$$
\begin{aligned}
x t_{2 p+1} X & \geq\left(p \alpha+p \beta+p^{2} \operatorname{diam} X\right) /\left(\begin{array}{c}
2 p+1 \\
2
\end{array}\right) \\
& =p\left(e_{x_{1} x_{2}}\left(x_{0}\right)+(p+1) \operatorname{diam} X\right) /\left(\begin{array}{c}
2 p+1 \\
2
\end{array}\right) \\
& \geq \frac{p+1}{2 p+1} \operatorname{diam} X+\frac{1}{2 p+1} \operatorname{exc} X,
\end{aligned}
$$

where the excess of $X, \operatorname{exc} X$, is defined as in [GP4] by

$$
\operatorname{exc} X=\min _{y_{1}, y_{2}} \max _{x} e_{y_{1} y_{2}}(x) \text {. }
$$

From (1.5) and (1.11) we conclude that if $x t_{q} X$ is minimal for some odd $q$, then $\operatorname{exc} X=0$. The same conclusion does not hold for even $q$. To see this let $X$ be the graph obtained from $V_{3}(\theta)$ in 1.6 (iii) by shortening one of the edges to less than one third of its original length. The 4-extent of this space is minimal although its excess is nonzero.

Now suppose $x t X$ is almost minimal, i.e., $x t X \leq \frac{1}{2} \operatorname{diam} X+\varepsilon$, for some small $\varepsilon>0$. Then by (1.11) and (1.3) we get

$$
\begin{aligned}
\operatorname{exc} X & \leq(2 p+1)\left[\frac{2 p+1}{2 p}\left(\frac{1}{2} \operatorname{diam} X+\varepsilon\right)-\frac{p+1}{2 p+1} \operatorname{diam} X\right] \\
& =\frac{1}{4 p} \operatorname{diam} X+\left(2 p+2+\frac{1}{2 p}\right) \varepsilon, \text { all } p .
\end{aligned}
$$

An analysis of the right-hand side of this inequality leads to the estimate

$$
\operatorname{exc} X \leq 10 \sqrt{\operatorname{diam} X} \sqrt{\varepsilon}
$$

for $\varepsilon \leq 0.5$ diam $X$. In particular,

Proposition 1.12. Let $X$ be a compact metric space. For any $\varepsilon>0$ there is $a$ $\delta>0$ such that exc $X<\varepsilon \cdot \operatorname{diam} X$ if $x t X \leq\left(\frac{1}{2}+\delta\right) \operatorname{diam} X$.

The converse of 1.12 does not hold as the following example shows.

Example 1.13. Fix $\theta \in\left(\frac{3}{8} \pi, \frac{1}{2} \pi\right]$ and let $C(\theta)$ be the region in $S_{1}^{2}$ bounded by two geodesics $c_{0}, c_{1}:[0, \pi] \rightarrow S_{1}^{2}$ making an angle $\theta$ at $c_{0}(0)=c_{1}(0)$. Let $X$ be the inner metric space obtained by identifying the $c_{0}$ 's in three copies of $C(\theta)$. In other words, $X$ is the spherical suspension of $V_{3}(\theta)$ from 1.6 (iii), or equivalently the warped product $X=V_{3}(\theta) \times{ }_{\sin }[0, \pi]$. Note that $V_{3}(\theta)$ is totally geodesic in $X$. Now clearly $x t_{3} X \geq x t_{3} V_{3}(\theta)=2 \theta$ and by $(1.3)$ $x t X \geq \frac{4}{3} \theta>\frac{\pi}{2}=\frac{1}{2} \operatorname{diam} X$. However, exc $X=0$.

\section{Positively curved spaces with large extents}

All metric spaces $(X, d i s t)$ considered from now on are assumed to be Alexandrov spaces. 
Before turning our attention to positively curved spaces we recall some basic general facts and notions:

First, if $c_{1}:\left[0, l_{1}\right] \rightarrow X$ and $c_{2}:\left[0, l_{2}\right] \rightarrow X$ are segments with $c_{1}(0)$ $=c_{2}(0)=p_{0} \in X$, the triple $\left(p_{0}, c_{1}, c_{2}\right)$ or simply $\left(c_{1}, c_{2}\right)$ is referred to as a hinge at $p_{0}$. Let $\bar{\alpha}_{k}\left(t_{1}, t_{2}\right)$ be the angle at $\bar{p}_{0} \in S_{k}^{2}$ in the comparison triangle $\left(\bar{p}_{0}, \overline{c_{1}\left(t_{1}\right)}, \overline{c_{2}\left(t_{2}\right)}\right)$ in $S_{k}^{2}$, i.e., $\operatorname{dist}\left(\bar{p}_{0}, \overline{c_{i}\left(t_{i}\right)}\right)=t_{i}, i=1,2$, and $\operatorname{dist}\left(c_{1}\left(t_{1}\right), c_{2}\left(t_{2}\right)\right)=\operatorname{dist}\left(\overline{c_{1}\left(t_{1}\right)}, \overline{c_{2}\left(t_{2}\right)}\right)$. From curv $X \geq k$ it follows that

$$
\bar{\alpha}_{k}\left(t_{1}, t_{2}\right) \text { is nonincreasing in } t_{1} \text { and } t_{2} \text {. }
$$

This allows one to define the angle between $c_{1}$ and $c_{2}$ by (cf. also [R])

$$
\varangle\left(c_{1}, c_{2}\right)=\lim \bar{\alpha}_{k}\left(t_{1}, t_{2}\right), t_{1}, t_{2} \rightarrow 0 .
$$

Now fix $p \in X$. A germ of segments emanating from $p$ is called a geodesic direction at $p$. The angle between segments emanating from $p$ defines a metric on the set $G_{p}$ of geodesic directions at $p$. The metric completion $S_{p}=\bar{G}_{p}$ of $G_{p}$ is called the space of directions at $p$. If $\operatorname{dim} X=n$, then $S_{p}$ is an $(n-1)$-dimensional Alexandrov space with curvature $\geq 1$ (see [BGP] or [P1]). Similarly, the infinite euclidean cone on $S_{p}$ is called the tangent space at $p$, and will be denoted by $T_{p} X$. The metric on $T_{p} X=S_{p} \times[0, \infty) / S_{p} \times\{0\}=C_{0} S_{p}$ is determined by the condition that the cone on any segment of length $\theta$ in $S_{p}$ is isometric to the planar euclidean cone of an arc of length $\theta$ in the unit circle $S^{1} \subset \mathbb{R}^{2}=S_{0}^{2}$. With this metric, $T_{p} X$ is an $n$-dimensional Alexandrov space with $\operatorname{curv} T_{p} X \geq 0$ ([Pl], [BGP]). Moreover, when given the induced length metric, the set of points of distance 1 from the vertex $p=S_{p} \times\{0\} \in T_{p} X$ is isometric to $S_{p}$. It is also worth pointing out that $T_{p} X$ is isometric to the Gromov-Hausdorff limit of the pointed spaces $(\lambda X, p), \lambda \rightarrow \infty$ (cf. [BGP]).

We say that a point $p \in X$ is euclidean if $T_{p} X$ is isometric to $\mathbb{R}^{n}=S_{0}^{n}$ (equivalently, $S_{p}$ is isometric to $S_{1}^{n-1}$ ). The following result about euclidean points is very useful.

Theorem 2.3 ([BGP], [Pl]). The set of euclidean points in an Alexandrov space is a dense $G_{\delta}$, i.e., a countable intersection of open dense subsets.

As for riemannian manifolds with a lower bound on sectional curvature, the following global comparison theorem provides the single most important tool.

Theorem 2.4. Let $X$ be an $n$-dimensional Alexandrov space, $n \geq 2$, with curv $X$ $\geq k$. Then

(i) For any triple $\left(p_{0}, p_{1}, p_{2}\right)$ in $X$ (with $3 x t_{3}\left(p_{0}, p_{1}, p_{2}\right)<2 \pi / \sqrt{k}$ if $k>0$ ) there is a unique (up to isometry) triple $\left(\bar{p}_{0}, \bar{p}_{1}, \bar{p}_{2}\right)$ in $S_{k}^{2}$ with $\operatorname{dist}\left(p_{i}, p_{j}\right)=$ $\operatorname{dist}\left(\bar{p}_{i}, \bar{p}_{j}\right)$. Moreover, for any segment $c_{0}$ from $p_{1}$ to $p_{2}$ and $0 \leq t \leq$ $\operatorname{dist}\left(p_{1}, p_{2}\right)$

$$
\operatorname{dist}\left(p_{0}, c_{0}(t)\right) \geq \operatorname{dist}\left(\bar{p}_{0}, \bar{c}_{0}(t)\right),
$$

where $\bar{c}_{0}$ is the unique segment from $\bar{p}_{1}$ to $\bar{p}_{2}$. Equivalently, the global version of (2.1) reads 
$\left(\mathrm{i}^{\prime}\right)$ For any hinge $\left(p_{0}, c_{1}, c_{2}\right)$ in $X$ with $0<\varangle\left(c_{1}, c_{2}\right)<\pi$ (and length $\left(c_{i}\right)<\frac{\pi}{\sqrt{k}}, i=1,2$, if $\left.k>0\right)$ we have

$$
\operatorname{dist}\left(c_{1}\left(l_{1}\right), c_{2}\left(l_{2}\right)\right) \leq \operatorname{dist}\left(\bar{c}_{1}\left(l_{1}\right), \bar{c}_{2}\left(l_{2}\right)\right),
$$

where $\left(\bar{p}_{0}, \bar{c}_{1}, \bar{c}_{2}\right)$ is the corresponding hinge in $S_{k}^{2}$, i.e., $\varangle\left(\bar{c}_{1}, \bar{c}_{2}\right)=\varangle\left(c_{1}, c_{2}\right)$.

(ii) If equality holds in (*) for some $0<t_{0}<\operatorname{dist}\left(p_{1}, p_{2}\right)$ and $c_{t_{0}}$ is a segment from $p_{0}$ to $c_{0}\left(t_{0}\right)$, then $c_{t_{0}}(s), 0<s \leq d i s t\left(p_{0}, c_{0}\left(t_{0}\right)\right)$, is joined to $p_{1}$ and $p_{2}$ by unique segments. Moreover, these segments together with their limit segments from $p_{0}$ to $p_{1}$ and $p_{2}$ form a surface isometric to the triangular surface in $S_{k}^{2}$ with vertices $\bar{p}_{0}, \bar{p}_{1}, \bar{p}_{2}$ and whose interior is totally geodesic in $X$.

(ii') If equality holds in $\left(*^{\prime}\right)$, then $\left(c_{1}, c_{2}\right)$ spans a surface isometric to the triangular surface in $S_{k}^{2}$ spanned by $\left(\bar{c}_{1}, \bar{c}_{2}\right)$, and with totally geodesic interior. In fact, any such surface is determined uniquely by a segment in $X$ between interior points of $c_{1}$ and $c_{2}$.

Part (i) of this theorem was proved in [BGP] and in [Pl]. The rigidity parts (ii), (ii $\left.{ }^{\prime}\right)$ will be proved in the Appendix. There are other equivalent versions of (i) and (i') in Theorem 2.4 like, e.g., the angle version customary to riemannian geometry. Since the main topic in this section is positively curved spaces, we point out some simple special consequences for this class.

Remark 2.5. Suppose $X$ is an $n$-dimensional Alexandrov space with curv $X \geq$ 1. Then $\operatorname{diam} X \leq \pi$ and $x t_{3} X \leq \frac{2 \pi}{3}$. Moreover, any geodesic triangle in $X$ with perimeter $2 \pi$ is degenerate, i.e., it is either a biangle or a closed geodesic in which antipodal points have distance $\pi$ (cf. [BGP]). If $\operatorname{dist}(p, q)=\operatorname{diamX}=$ $\pi$, then $\operatorname{exc} X=0$. In fact any $x \in X-\{p, q\}$ lies on a unique segment from $p$ to $q$. Moreover, $E=\left\{x \in X \mid \operatorname{dist}(p, x)=\operatorname{dist}(q, x)=\frac{\pi}{2}\right\}$ is a totally geodesic $(n-1)$-dimensional Alexandrov space with curv $E \geq 1$, and $X$ is isometric to the spherical suspension $\Sigma_{1} E$ of $E$ (cf. [GP4], [BGP], or 2.4 (i) and (ii)). The metric on $\Sigma_{1} E$ is determined by the requirement that the suspension of a segment of length $\theta$ in $E$ is isometric to the region in $S_{1}^{2}$ bounded by a biangle of angle $\theta$. Similarly half of $\Sigma_{1} E$, the spherical cone on $E$, is denoted by $C_{1} E$, i.e., $C_{1} E \cup_{E} C_{1} E=\Sigma_{1} E$. These are both special cases of the spherical join construction in Example 2.16.

It is convenient to adopt the following terminology and conventions from [GP3,5] to the setting of Alexandrov spaces. For $p \in X$ and a geodesic direction $v \in G_{p} \subset S_{p}$, let $c_{v}:\left[0, t_{v}\right] \rightarrow X$ be the unique maximal segment representing $v$ (geodesics cannot bifurcate in an Alexandrov space). The function $v \rightarrow t_{v}$ is upper semicontinuous and we define the segment domain $\operatorname{seg}(p)$ for $p$ to be the closure in $T_{p} X$ of the subset $\left\{(v, t) \mid 0 \leq t \leq t_{v}\right\}$. Note that if $u \in S_{p}-G_{p}$, then $t_{u}=0$ and $\exp _{p}: \operatorname{seg}(p) \rightarrow X$ given by $(t, v) \rightarrow c_{v}(t)$ is a well-defined continuous and surjective map.

To give a better description of the metric behavior of $\exp _{p}: \operatorname{seg}(p) \rightarrow X$ we change the metric on $T_{p} X$ (or the ball $D\left(p, \frac{\pi}{\sqrt{k}}\right) \subset T_{p} X$ if $k>0$ ). By a unique radial change, all euclidean sectors are replaced by constant curvature 
$k$ sectors making the same angle at $p$. For $k \leq 0$ we denote $T_{p} X$ with this new metric as $C_{k} S_{p}$. If $k>0$, all points at distance $\frac{\pi}{\sqrt{k}}$ from $p \in T_{p} X$ are identified and the resulting metric space $\Sigma_{k} S_{p}$ is isometric to $\frac{1}{\sqrt{k}} \Sigma_{1} S_{p}$ (cf. 2.5). In any case we can view $\operatorname{seg}(p)$ as a subspace of $C_{k} S_{p}, k \leq 0$, or of $\Sigma_{k} S_{p}, k>0$ (cf. 2.5). When given the induced metric we have

$$
\exp _{p}: \operatorname{seg}(p) \rightarrow X \quad \text { is distance nonincreasing. }
$$

In fact, this statement is easily seen to be equivalent to the hinge version ( $\mathrm{i}^{\prime}$ ) of Theorem 2.4. Because of (2.3), we need to use (2.6) mostly for euclidean points $p \in X$. In this case, $T_{p} X$ has simply been replaced by $S_{k}^{n} \supset \operatorname{seg}(p)$.

It should be clear from the discussion above that positively curved Alexandrov spaces play a particularly significant role: They are exactly the spaces that can occur as spaces of directions in Alexandrov spaces.

In the remaining part of this section, we discuss the extremal problems for $q$-extents on Alexandrov spaces $X$ with curv $X \geq 1$. This can be interpreted in two ways related to either (7) (as in 2.7 below), or to (5) (as in 2.9-2.14 below):

In the first case we have the following general result, which implies Theorem A of the introduction.

Theorem 2.7. Let $X$ be an $n$-dimensional, $n \geq 2$, Alexandrov space with curv $X$ $\geq 1$. Then

$$
x t_{q} X \leq x t_{q} S_{1}^{n}
$$

for all $q \geq 2$. Moreover, equality holds for some $q$ if and only if diamX $=\pi$.

Proof. The inequality is an immediate consequence of 2.3 and (2.6) (cf. 2.5). Now suppose $x t_{q} X=x t_{q} S_{1}^{n}$ for some $q$, and let $\left(x_{1}, \cdots, x_{q}\right)$ be a $q$-extender in $X$. Choose a euclidean point $p \in X$ (cf. 2.3) and let $\left(\bar{x}_{1}, \cdots, \bar{x}_{q}\right)$ be a $q$-tuple in $\operatorname{seg}(p) \subset S_{1}^{n}$ with $\exp _{p} \bar{x}_{i}=x_{i}, i=1, \cdots, q$. By assumption and (2.6)

$$
x t_{q}\left(S_{1}^{n}\right)=x t_{q}(X)=x t_{q}\left(x_{1}, \cdots, x_{q}\right) \leq x t_{q}\left(\bar{x}_{1}, \cdots, \bar{x}_{q}\right) \leq x t_{q}\left(S_{1}^{n}\right) .
$$

Thus $\left(\bar{x}_{1}, \cdots, \bar{x}_{q}\right)$ is a $q$-extender in $S_{1}^{n}$ and $\operatorname{dist}\left(\bar{x}_{i}, \bar{x}_{j}\right)=\operatorname{dist}\left(x_{i}, x_{j}\right)$ for all $i, j$. From the description of all possible $q$-extenders in $S_{1}^{n}$ given in Theorem 1.8, either $\operatorname{dist}\left(\bar{x}_{i}, \bar{x}_{j}\right)=\pi$ for some pair, or $\operatorname{dist}\left(\bar{x}_{i}, \bar{x}_{j}\right)+\operatorname{dist}\left(\bar{x}_{j}, \bar{x}_{k}\right)+$ $\operatorname{dist}\left(\bar{x}_{k}, \bar{x}_{i}\right)=2 \pi$ for some triple in $\left(\bar{x}_{1}, \cdots, \bar{x}_{q}\right)$. Since the same conclusion holds for the corresponding points in $X, 2.5$ completes the proof.

We mention that in analogy to the diameter sphere theorem [GS], it follows from Perelman's work [P] that if $\operatorname{diam} X \geq x t_{q} X>\frac{\pi}{2}$, then $X$ is homeomorphic to a spherical suspension $\Sigma_{1} E$.

Note that if in the second case where $x t_{q} X$ is maximal, i.e., $x t_{q} X=\operatorname{diam} X$, so is $x t_{q^{\prime}} X, q^{\prime} \leq q$. It is therefore immediate from 2.4 (i) that if $\operatorname{diam} X>\frac{\pi}{2}$, then no $x t_{q} X$ is maximal, $q \geq 3$. With this in mind we now focus our attention on Alexandrov spaces $X$ with $\operatorname{curv} X \geq 1$ and $\operatorname{diam} X=\frac{\pi}{2}$ : 
Problem 2.8. Describe all $n$-dimensional Alexandrov spaces $X$ with $\operatorname{curv} X \geq$ 1 and $\operatorname{diam} X=\frac{\pi}{2}$.

When restricted to riemannian manifolds an essentially complete metric classification was given in [GG]. The general problem seems very hard (cf. [GP6]). Here we will give a complete metric classification under the additional assumption $x t_{n+1} X=\frac{\pi}{2}$.

We begin with the following simple illustration of how to apply the rigidity parts of the comparison Theorem 2.4.

Lemma 2.9. Let $X$ be an Alexandrov space with curv $X \geq 1$ and diam $X=\frac{\pi}{2}$. Then $x t_{q+1} X=\frac{\pi}{2}, q \geq 1$, if and only if the right angled spherical $q$-simplex $\Delta^{q}=C_{1} \Delta^{q-1}=\cdots=C_{1}^{q}$ (point) $\subset S_{1}^{q}$ is isometrically embedded in $X$, with totally geodesic interior.

Proof. Pick a $(q+1)$-extender $\left(x_{0}, \cdots, x_{q}\right)$ for $X$. By assumption $\operatorname{dist}\left(x_{i}, x_{j}\right)$ $=\frac{\pi}{2}$ for all $i \neq j$. We construct the desired simplex in $q$ steps. First join $x_{0}$ and $x_{1}$ by a segment, $\Delta^{1}=\left[0, \frac{\pi}{2}\right]=C_{1}$ (point). Note that all points of $\Delta^{1}$ are at distance $\frac{\pi}{2}$ from each of $x_{2}, \cdots, x_{q}$ by 2.4(i) and $\operatorname{diam} X=\frac{\pi}{2}$. Now join, say, the midpoint $m_{01}$ of $\Delta^{1}$ to $x_{2}$ by a segment, and use it to sweep out a $\Delta^{2}$ via 2.4(ii). Again all points of $\Delta^{2}$ are at distance $\frac{\pi}{2}$ from each of $x_{3}, \cdots, x_{q}$. Now join $x_{3}$ to, e.g., the barycenter $m_{012}$ of $\Delta^{2}$ and sweep out triangular surfaces along all segments in $\Delta^{2}$ through $m_{012}$ using 2.4 (ii). The resulting cone on $\Delta^{2}$ is in fact isometric to $\Delta^{3}$. To see this from 2.4 (ii), it is important to observe that any segment from an interior point of the segment from $x_{3}$ to $m_{012}$ to any point of $\Delta^{2}$ is unique. Continuing this process $q$ times completes the construction. That the interior of $\Delta^{q}$ is totally geodesic follows from the fact that $\Delta^{q}$ is isometrically embedded, and that geodesics in $X$ cannot bifurcate.

Next we give an inductive method for generating examples of $n$-dimensional Alexandrov spaces $X$ with curv $X \geq 1$ and $\operatorname{diam} X=x t_{n+1} X=\frac{\pi}{2}$ :

Example 2.10. Suppose $\tilde{Y}$ is an $(n-1)$-dimensional Alexandrov space with $\operatorname{curv} \tilde{Y} \geq 1$ and $i: \tilde{Y} \rightarrow \tilde{Y}$ an isometric involution. Then $Y=\tilde{Y} / i$ is an $(n-1)$-dimensional Alexandrov space with $\operatorname{curv} Y \geq 1$ and $(\tilde{Y}, i)$ is referred to as a "2-fold" of $Y$. The spherical suspension $\tilde{X}=\Sigma_{1} \tilde{Y}$ is an $n$-dimensional Alexandrov space with $\operatorname{curv} \tilde{X} \geq 1$ and $I=-\Sigma i: \tilde{X} \rightarrow \tilde{X},\left(\tilde{y}, \frac{\pi}{2}+\theta\right) \rightarrow$ $\left(i(\tilde{y}), \frac{\pi}{2}-\theta\right), \tilde{y} \in \tilde{Y}, \theta \in\left[0, \frac{\pi}{2}\right]$, is an isometric involution. Thus also

$$
\begin{aligned}
X & =\tilde{X} / I=(\Sigma \tilde{Y} /-\Sigma i)=C_{1}(\tilde{Y} \rightarrow Y) \\
& =\text { the spherical mapping cone on } \tilde{Y} \rightarrow Y=\tilde{Y} / i
\end{aligned}
$$

is an $n$-dimensional Alexandrov space with curv $X \geq 1$. Moreover, if $x t_{n} Y=$ $\operatorname{diam} Y=\frac{\pi}{2}$, then $x t_{n+1} X=\operatorname{diam} X=\frac{\pi}{2}$.

To start the procedure, recall that by convention a 1-dimensional Alexandrov space $X$ has $\operatorname{curv} X \geq 1$ if and only if $\operatorname{diam} X \leq \pi$, i.e., $X$ is an interval of 
length $\leq \pi$ or a circle of length $\leq 2 \pi$. In particular

$$
X=\left\{\begin{aligned}
\text { (a) } \Delta^{1}= & {\left[0, \frac{\pi}{2}\right]=C_{1}(\text { poirt })=\text { the spherical cone on a point } } \\
\text { (b) } D \Delta^{1} & =\left[-\frac{\pi}{2}, \frac{\pi}{2}\right] /\left(-\frac{\pi}{2} \sim \frac{\pi}{2}\right)=\text { the double of } \Delta^{1} \\
& =R P_{1}^{1}=S^{1} /(-x \sim x)=\text { the real projective line }
\end{aligned}\right.
$$

is the complete list of 1-dimensional spaces with $\operatorname{diam} X=x t_{2} X=\frac{\pi}{2}$ and curv $X \geq 1$.

In order to apply the above method to construct 2-dimensional examples, we list all the possible 1-dimensional "2-folds" $(\tilde{Y}, i)$ of the spaces in (1):

$$
(\tilde{Y}, i)=\left\{\begin{array}{l}
(\tilde{\mathrm{a}})=\left\{\begin{aligned}
\text { (i) } & \left(\Delta^{1}, i d\right), \\
\text { (ii) } & \left(\left[-\frac{\pi}{2}, \frac{\pi}{2}\right],-i d\right), \\
\text { (iii) } & \left(D \Delta^{1},-i d\right),
\end{aligned}\right. \\
(\tilde{\mathrm{b}})=\left\{\begin{aligned}
\text { (i) } & \left(R P_{1}^{1}, i d\right), \\
\text { (ii) } & \left(S_{1}^{1},-i d\right) .
\end{aligned}\right.
\end{array}\right.
$$

This yields the following 2-dimensional examples:

$$
X=\left\{\begin{array}{l}
\Delta^{2}=\text { the right angled spherical 2-simplex } \\
\quad=C_{1}\left(\Delta^{1}\right),(\tilde{\mathrm{a}}, \mathrm{i}), \\
C_{1}\left(D \Delta^{1}\right)=C_{1}\left(R P^{1}\right),(\tilde{\mathrm{a}}, \mathrm{ii}),(\tilde{\mathrm{b}}, \mathrm{i}) \\
D \Delta^{2}=\text { the double spherical } 2 \text {-simplex } \\
\quad=\Delta^{2} \cup \Delta^{2} /\left(\partial \Delta^{2} \sim \partial \Delta^{2}\right),(\tilde{\mathrm{a}}, \mathrm{iii}), \\
R P^{2}=S_{1}^{2} /(-x \sim x)=\text { the real projective plane, }(\tilde{\mathrm{b}}, \mathrm{ii}) .
\end{array}\right.
$$

Our next result shows in particular that any $n$-dimensional Alexandrov space $X$ with curv $X \geq 1$ and $x t_{n+1} X=\operatorname{diam} X=\frac{\pi}{2}$ is generated by the method described in 2.10 .

Lemma 2.11. Assume $X$ is an $n$-dimensional Alexandrov space with $n \geq 2$, curv $X \geq 1$, and $\operatorname{diam} X=x t_{n+1} X=\frac{\pi}{2}$. Let $\left(x_{0}, \cdots, x_{n}\right)$ be an $(n+1)$ extender in $X$ and $A_{0}=\left\{x \mid\right.$ dist $\left.\left(x, x_{0}\right)=\frac{\pi}{2}\right\}$. Then $A_{0}$ is an $(n-1)$ dimensional Alexandrov space with curv $A_{0} \geq 1$ and $x t_{n} A_{0}=\operatorname{diam} A_{0}=\frac{\pi}{2}$. Moreover, $S_{x_{0}}$ is a 2-fold of $A_{0}$ and $X$ is isometric to $C_{1}\left(S_{x_{0}} \rightarrow A_{0}\right)$.

Proof. By distance comparison (cf. 2.4) $A_{0}$ is convex in $X$ and, obviously, curv $A_{0} \geq 1$ and $\operatorname{diam} A_{0}=x t_{n} A_{0}=\frac{\pi}{2}$. Moreover, we claim that $\operatorname{dim} A_{0}=$ $n-1$. In fact $A_{0} \supset \Delta^{n-1}$ by 2.9 , and a space of directions argument shows that $A_{0}$ cannot have dimension $n$ (cf., e.g., 2.3 and 2.4). Using 2.4 (ii') and 
2.4(ii) (as in 2.9), we see that each segment from $x_{0}$ to $y \in A_{0}$, together with each $\Delta^{n-1} \subset A_{0}$ containing $y$, determines a unique spherical $n$-simplex $\Delta^{n}$. Moreover, a simple space of directions argument shows that there can be at most two such $\Delta^{n}$ 's with common $\Delta^{n-1}$. We claim that $X$ is the union of all these $n$-simplices, and thus in particular that any $x \in X-\left(A_{0} \cup x_{0}\right)$ lies on a unique segment from $x_{0}$ to $A_{0}$ : If not, let $y$ be a point in the union of simplices closest to $x$. Now using that $\operatorname{diam} X=\frac{\pi}{2}$ it is not difficult to see that any segment from $x$ to $y$ will be perpendicular to all segments emanating from $y$ in one of these simplices. Since this set of directions at $y$ has nonempty interior we get a contradiction.

All of this shows in particular that $S_{x_{0}}=G_{x_{0}}$, and there is a well-defined map $E: S_{x_{0}} \rightarrow A_{0}$, given by $E(v)=c_{v}\left(\frac{\pi}{2}\right), v \in S_{x_{0}}$. Moreover, $E$ is at most 2-1 and the restriction of $E$ to the set of directions $\Delta^{n-1} \subset S_{x_{0}}$ corresponding to a $\Delta^{n} \subset X$ is an isometry. If $E$ is $1-1$, then $X=C_{1} A_{0}$. If $E$ is not $1-1$, then $i: S_{x_{0}} \rightarrow S_{x_{0}}$, defined by $i(v)=\bar{v}$ if $E(v)=E(\bar{v})$ and $v \neq \bar{v}$ and $i(v)=v$ if $E^{-1}(E(v))=\{v\}$, is an isometric involution. It follows that $\left(S_{x_{0}}, i\right)$ is a " 2 fold" of $A_{0}$ and $X=C_{1} S_{x_{0}} /\left(\left(v, \frac{\pi}{2}\right) \sim\left(i(v), \frac{\pi}{2}\right)\right)$ is isometric to the quotient of the spherical suspension $\Sigma_{1} S_{x_{0}}$ by the isometric involution $I: \Sigma_{1} S_{x_{0}} \rightarrow \Sigma_{1} S_{x_{0}}$ given by $I\left(v, \frac{\pi}{2}+\theta\right)=\left(i(v), \frac{\pi}{2}-\theta\right), \theta \in\left[0, \frac{\pi}{2}\right]$. This completes the proof of 2.11 .

To obtain a classification of all Alexandrov spaces $X$ with curv $X \geq 1$ and $x t_{\operatorname{dim} X+1} X=\operatorname{diam} X=\frac{\pi}{2}$ via 2.10 and 2.11 , we need an inductive procedure to construct all possible 2-folds.

Lemma 2.12. Let $X$ be an $n$-dimensional Alexandrov space with curv $X \geq 1$ and $x t_{n+1} X=\operatorname{diam} X=\frac{\pi}{2}$. If $(\tilde{X}, I)$ is a nontrivial 2-fold of $X$, there is an $(n-1)$ dimensional Alexandrov space $Y$ with $x t_{n} Y=\operatorname{diam} Y=\frac{\pi}{2}$ and a 2-fold $(\tilde{Y}, i)$ of $Y$ such that

$$
\tilde{X}=\Sigma \tilde{Y} \text { and } I=-\Sigma i
$$

or

(ii) $\quad \tilde{X}=\Sigma \tilde{Y} /-\Sigma i$ and $I$ is induced from $\Sigma j$, where $j: \tilde{Y} \rightarrow \tilde{Y}$ is an isometric involution commuting with $i$ and inducing a 2-fold on $Y$, i.e., $x t_{n} \tilde{Y} /\langle i, j\rangle=\frac{\pi}{2}$.

Proof. Fix an $(n+1)$-extender $\left(x_{0}, \cdots, x_{n}\right)$ in $X$ and consider the quotient $\operatorname{map} \pi: \tilde{X} \rightarrow X$.

(i) If, say, $\pi^{-1}\left(x_{0}\right)$ consists of two points, it is easy to see that they are at distance $\pi$ apart, and that $\tilde{X}=\Sigma \tilde{A}_{0}$, where $\tilde{A}_{0}=\pi^{-1}\left(A_{0}\right)$ and $I=-\Sigma i$, where $i=I \mid \tilde{A}_{0}$.

(ii) Now suppose $\pi^{-1}\left(x_{i}\right)$ consists of only one point for $i=0, \cdots, n$. As above, $\tilde{A}_{0}=\pi^{-1}\left(A_{0}\right)$ is convex and an easy induction argument shows that $x t_{n+1} \tilde{X}=\operatorname{diam} \tilde{X}=\frac{\pi}{2}$ (cf. proof of 2.11). Set $\tilde{Y}=S_{\tilde{x}_{0}}$, where $\tilde{x}_{0}=\pi^{-1}\left(x_{0}\right)$. 
Then by 2.11, $\tilde{X}=\Sigma \tilde{Y} /-\Sigma i$, for some isometric involution $i: S_{\tilde{x}_{0}} \rightarrow S_{\tilde{x}_{0}}$ and $\tilde{A}_{0}=S_{\tilde{x}_{0}} / i=Y$. Clearly $I$ induces an isometric involution $j: S_{\dot{x}_{0}} \rightarrow S_{\tilde{x}_{0}}$ which commutes with $i$ and whose induced map on $\tilde{A}_{0}=S_{\tilde{x}_{0}} / i$ is $I \mid \tilde{A}_{0}$. In particular, $I: \Sigma \tilde{Y} /-\Sigma i \rightarrow \Sigma \tilde{Y} /-\Sigma i$ is induced from $\Sigma j: \Sigma \tilde{Y} \rightarrow \Sigma \tilde{Y}$.

Starting with the complete classification of our spaces and their 2-folds in dimension 1 (Example 2.10), and using 2.12 and 2.11, we obtain a complete classification in all dimensions. Again using 2.11 and 2.12 and an easy induction argument it is in fact not difficult to give the following more satisfactory description.

Theorem 2.13. Let $G=\left(\mathbb{Z}_{2}\right)^{n+1}$ be the group generated by all reflections in coordinate hyperplanes in $\mathbb{R}^{n+1}$. Then $X$ is an n-dimensional Alexandrov space with curv $X \geq 1$ and $x t_{n+1} X=\operatorname{diam} X=\frac{\pi}{2}$ if and only if it is isometric to $S_{1}^{n} / H$, where $H \subset G$ is a subgroup acting without fixed points. Moreover, for any 2-fold $(\tilde{X}, I)$ of $X$, there is a subgroup $H^{\prime} \subset H$ and an $h \in H$ such that $H=H^{\prime} \oplus\langle h\rangle, \tilde{X}=S_{1}^{n} / H^{\prime}$, and $I$ is induced by $h$.

To prove Corollary $\mathrm{C}$ from the introduction we need the following observation.

Theorem 2.14. If an n-dimensional Alexandrov space $X$ with curv $X \geq 1$ and $x t_{n+1} X=$ diam $X=\frac{\pi}{2}$ is a (generalized) manifold, it is either isometric to $R P_{1}^{n}$ or homeomorphic to $S^{n}$.

Proof. First we use the description $X=C_{1}(\tilde{Y} \rightarrow Y)$ from 2.11. By Van Kampen's theorem $\pi_{1}(X)=\pi_{1}(Y) / N\left(i m \pi_{1}(\tilde{Y})\right)$. An easy induction argument then gives that $\pi_{1}(X) \neq\{1\}$ if and only if $X$ is isometric to $R P_{1}^{n}$ (the only nontrivial 2-fold of $R P_{1}^{n}$ is $S_{1}^{n}$ ).

Assume from now on that $X \neq R P_{1}^{n}$, i.e., in particular $\pi_{1}(X)=\{1\}$. The Mayer-Vietoris sequence applied to $X=C \tilde{Y} \cup Y$ yields

$$
\begin{aligned}
0 \rightarrow H_{n}(X) & \rightarrow H_{n-1}(\tilde{Y}) \rightarrow H_{n-1}(Y) \rightarrow H_{n-1}(X) \rightarrow H_{n-2}(\tilde{Y}) \rightarrow \cdots \\
& \cdots \rightarrow H_{2}(X) \rightarrow H_{1}(\tilde{Y}) \rightarrow H_{1}(Y) \rightarrow 0 .
\end{aligned}
$$

Now suppose $X$ is a generalized manifold. Then in particular $H_{\star}(\tilde{Y})=$ $H_{\star}\left(S^{n-1}\right)$, and consequently $H_{q}(Y) \cong H_{q}(X)$ for $q \leq n-2$ and, by Poincaré duality, $H_{n-1}(X)=\{0\}$. If we use homology with $\mathbb{Z}_{2}$-coefficients, we deduce moreover that $H_{n}(X) \rightarrow H_{n-1}(\tilde{Y})$ is an isomorphism and hence $H_{n-1}(Y)=$ $\{0\}$. From [GP6], therefore, we know that $\partial \tilde{Y} \neq \varnothing$, which since curv $Y \geq$ $1>0$ implies that $Y$ is contractible. In fact by the soul theorem of [P1], $Y$ is homeomorphic to the cone of the space of directions, $S_{p_{0}}$, where $p_{0} \in Y$ is the unique point at maximal distance from $\partial Y$. All in all we conclude that $H_{\star}(X)=H_{\star}\left(S^{n}\right)$, which for $n \neq 3$ suffices by the solution of the generalized Poincaré conjecture. For $n=3$ a direct argument based on 2.10, 2.11, and 2.12 can be given. Alternatively we can use the description $X=S_{1}^{n} / H$ from 2.12: 
First note that $H$ acts freely on $S_{1}^{n}$ if and only if $H=\mathbb{Z}_{2}$ and $X=R P_{1}^{n}$.

Now suppose $X \neq R P_{1}^{n}$ is a generalized manifold, and let $p \in S_{1}^{n}$ with non-trivial isotropy group $H_{p} \subset H$. If $[p] \in X$ denotes the orbit of $p$, then clearly $\tilde{Y}=S_{[p]}=\Sigma^{l}\left(S_{1}^{n-l-1} / H_{p}\right)=S_{1}^{l-1} *\left(S_{1}^{n-l-1} / H_{p}\right)$, where $l=\operatorname{dimFix} H_{p}$ and $S_{1}^{n-\ell-1}$ is the normal sphere at $\mathrm{p}$ to fix $H_{p}$. Since by assumption $H_{\star}(\tilde{Y})=$ $H_{\star}\left(S^{n-1}\right)$, we see that $H_{\star}\left(S_{1}^{n-\ell-1} / H_{p}\right)=H_{\star}\left(S_{1}^{n-\ell-1}\right)$. By induction we may assume that $S_{1}^{n-\ell-1} / H_{p}$ is homeomorphic to $S^{n-\ell-1}$, and hence $\tilde{Y}$ is homeomorphic to $S^{n-1}$. By the same argument, any space of directions of $\tilde{Y}$ is homeomorphic to $S^{n-2}$. Now since $\tilde{Y}$-Fix $i$ is dense in $\tilde{Y}$, an easy convexity argument in $Y$ shows that $\pi$ (Fix $i)=\partial Y \neq \varnothing$, where $\pi: \tilde{Y} \rightarrow Y=\tilde{Y} / i$ is the quotient map. It follows that $(\tilde{Y}, i)$ is the double of $Y$ and in particular $S_{p_{0}}=S_{\tilde{p}_{0}}$ for some $\tilde{p}_{0} \in \tilde{Y}$-Fix $i$. We conclude that $Y$ is homeomorphic to $D^{n-1}$ and $X$ is homeomorphic to $\Sigma \tilde{Y} /-\Sigma i \sim S_{1}^{n} / I \sim S^{n}$, where $I$ is the isometric involution with Fix $I=S_{1}^{n-2}$.

Remark 2.15. In the above proof it actually follows that either $\tilde{E}=S_{1}^{n-\ell-1} / H_{p}$ is the double of $E=\tilde{E} / i \sim D^{n-\ell-1}$ and therefore $\ell=0$, or $i \mid \tilde{E}=i d$ and then $\ell=1(n \geq 3)$. This observation allows us to sharpen the conclusion of 2.13. In fact by induction it can be seen that if $X \neq R P_{1}^{n}$ is an $n$-dimensional Alexandrov space with curv $X \geq 1$ and $x t_{n+1} X=\operatorname{diam} X=\frac{\pi}{2}$, then $X$ is a generalized manifold if and only if it is isometric to a space of the form $D\left(\Delta^{n_{1}}\right) * D\left(\Delta^{n_{2}}\right) * \cdots * D\left(\Delta^{n_{r}}\right)$ where $n_{1}+\cdots+n_{r}+(r-1)=n$ (cf. 2.16 and 2.17 below).

We conclude this section by pointing out that the spaces in Theorem 2.13 generate an interesting group. This is related to the following construction of positively curved spaces.

Example 2.16 (The spherical join construction). Let $X$ and $Y$ be Alexandrov spaces. The product $X \times Y$ admits a natural Alexandrov structure. Moreover, if $p \in X, q \in Y$, then $S_{(p, q)}=S_{p} * S_{q}=S_{p} \times C S_{q} \cup C S_{p} \times S_{q}$, the topological join of $S_{p}$ and $S_{q}$. In particular we see that if $X$ and $Y$ are Alexandrov spaces with curv $X \geq 1$ and curv $Y \geq 1$, then $X * Y$ has a natural Alexandrov structure with curv $X * Y \geq 1 \quad\left(X * Y=S_{(p, q)}\left(C_{0} X \times C_{0} Y\right)\right)$. Since $\operatorname{diam} X * Y \geq \frac{\pi}{2}$, the subclass of spaces $X$ with curv $X \geq 1$ and $\operatorname{diam} X \geq \frac{\pi}{2}$ is especially interesting. Another much smaller class is provided by the class of Alexandrov spaces $X$ with curv $X \geq 1$ and $\operatorname{diam} X=x t_{\operatorname{dim} X+1} X=\frac{\pi}{2}$. It is not difficult to check (using a more detailed description of the metric as in [GP6]) that this class is closed under the spherical join operation. This leads to the following

Problem 2.17. Under suitable equivalence between spaces, determine the Grothendieck group corresponding to the abelian semigroup $(\mathscr{G}, *)$ where $\mathscr{G}$ consists of $n$-dimensional spaces $X$ with $\operatorname{curv} X \geq 1$ and $x t_{n+1} X=\operatorname{diam} X=$ $\frac{\pi}{2}, n=0,1,2, \cdots$.

For other classes of positively curved spaces that are closed under spherical join see [GP 6]. 


\section{AlEXANDROV MANIFOldS WITH BOUNDED RADIUS AND LARGE PACKING RADII}

Let $X$ be a compact Alexandrov space. It is immediate from (2.6) that pack $_{q} X \leq \operatorname{pack}_{q} \operatorname{seg}(p)$ for any $p \in X$ and integer $q \geq 2$. If in particular $\operatorname{dim} X=n$, curv $X \geq k$, and $\operatorname{rad} X \leq R$, then

$$
\operatorname{pack}_{q} X \leq \operatorname{pack}_{q} D_{k}^{n}(R),
$$

where we have used 2.3 as well.

Noting that $\operatorname{pack}_{q} D_{k}^{n}(R)$ is obviously increasing in $n$, we see from (3.1) that if $X$ is an Alexandrov space with $\operatorname{curv} X \geq k$ and $\operatorname{rad} X \leq R$, then $\operatorname{dim} X>n$ if pack $_{q} X>$ pack $_{q} D_{k}^{n}(R)$ for some $q$. If in particular we consider the class of all closed riemannian $n$-manifolds $M$ with $\sec M \geq k, \operatorname{rad} M \leq R$, and $\operatorname{pack}_{q} M \geq \operatorname{pack}_{q} D_{k}^{n-1}(R)+\varepsilon$ for some fixed $q$ and $\varepsilon>0$, then any Alexandrov limit space $X$ has curv $X \geq k, \operatorname{rad} X \leq R, \operatorname{pack}_{q} X \geq \operatorname{pack}_{q} D_{k}^{n-1}(R)+\varepsilon$, and $\operatorname{dim} X=n$, by [GP3] and the above. Thus no collapse can occur in this class. Consequently there is a $v=v(\varepsilon, n, k, R, q)>0$ such that all of these manifolds have vol $M \geq v$. From [GPW] (if $n \neq 3$ ) and [P] in general it follows that this class contains at most finitely many topological types. The first statement in Corollary $\mathrm{E}$ of the introduction is a special case.

Since $D_{k}^{n}(R)$ is in itself an Alexandrov space (unless $k>0$ and $R>\frac{\pi}{2 \sqrt{k}}$ ), the above inequality is obviously optimal for Alexandrov spaces in general. Our main purpose in this section is to investigate the optimality question for (3.1) when $X$ in addition is assumed to be a (generalized) manifold.

Let us first consider $n$-dimensional Alexandrov spaces $X$ with curv $X \geq$ $k, k<1 / 4$, and radius normalized by $\operatorname{rad} X=\pi$. In view of (3.1) the following problem is of obvious interest.

Problem 3.2. Determine $\operatorname{pack}_{q} D_{k}^{n}(\pi)$ and the corresponding packers, for all $q, n$, and $k<\frac{1}{4}$. For $q$ large this is equivalent to the classical packing problem.

The solution to this problem for $q \leq n+1$ provides the starting point for our discussion: A $q$-packer in $D_{k}^{n}(\pi), k<\frac{1}{4}$ and $q \leq n+1$, consists of the vertices in the unique (up to isometry) regularly inscribed $(q-1)$-simplex $\Delta_{k}^{q-1}$ in $D_{k}^{q-1} \subset D_{k}^{n}$. To see this first note that any $q$-packer in $D_{k}^{n}(\pi)$ must be contained in $\partial D_{k}^{q-1}(\pi)$, and then use the characterization of $q$-packers in $S_{1}^{q-2}$ described in Proposition 2.4 of [GW] (cf. also [GM]). We begin with an analogue of 2.8 .

Lemma 3.3. Let $X$ be an $n$-dimensional Alexandrov space with curv $X \geq k, k<$ $\frac{1}{4}, \operatorname{rad} X=\pi$, and pack $_{n+1} X=$ pack $_{n+1} D_{k}^{n}(\pi)$. Then $X$ contains an isometric copy of $\Delta_{k}^{n}$ with totally geodesic interior. Moreover, if $m$ is the barycenter of $\Delta_{k}^{n} \subset X$, then $X=D(m, \pi)$.

Proof. Let $m \in X$ be the center of a ball with radius $\pi$ and $D(m, \pi)=X$. Choose an $(n+1)$-packer $\left(x_{0}, \cdots, x_{n}\right)$ for $X$ and let $\left(\bar{x}_{0}, \cdots, \bar{x}_{n}\right)$ be points 
in $\operatorname{seg}(m)$ such that $\exp _{m} \bar{x}_{i}=x_{i}, i=0, \cdots, n$. We denote directions corresponding to $\left(\bar{x}_{0}, \cdots, \bar{x}_{n}\right)$ by $v_{0}, \cdots, v_{n} \in G_{m} \subset S_{m}$. Now pick a euclidean point $u \in S_{m}$ (cf. 2.3), and let $\bar{v}_{0}, \cdots, \bar{v}_{n} \in \operatorname{seg}(u) \subset S_{1}^{n-1}$ be points such that $\exp _{u} \bar{v}_{i}=v_{i}, i=0, \cdots, n$. By $(2.6) \varangle\left(\bar{v}_{i}, \bar{v}_{j}\right) \geq \varangle\left(v_{i}, v_{j}\right)$ for all $i, j$. In $S_{k}^{n}$ viewed as $C_{k} S_{1}^{n-1}$ (or $\Sigma_{k} S_{1}^{n-1}$ if $k>0$ ) consider the points $\overline{\bar{x}}_{i}$, at distance $\operatorname{dist}\left(m, x_{i}\right)$ from the cone (or vertex) point of $S_{k}^{n}$ in direction $\bar{v}_{i}, i=0, \cdots, n$. Then $\overline{\bar{x}}_{0}, \cdots, \overline{\bar{x}}_{n} \in D_{k}^{n}(\pi)$ and $\operatorname{dist}\left(\overline{\bar{x}}_{i}, \overline{\bar{x}}_{j}\right) \geq$ $\operatorname{dist}\left(x_{i}, x_{j}\right)$ for all $i, j$ by 2.4 (ii) since $\varangle\left(\bar{v}_{i}, \bar{v}_{j}\right) \geq \varangle\left(v_{i}, v_{j}\right)$. By assumption this means that $\left(\overline{\bar{x}}_{0}, \cdots, \overline{\bar{x}}_{n}\right)$ is an $(n+1)$-packer in $D_{k}^{n}(\pi)$ and moreover $\operatorname{dist}\left(x_{i}, x_{j}\right)=\operatorname{dist}\left(\overline{\bar{x}}_{i}, \overline{\bar{x}}_{j}\right)$ and hence $\varangle\left(\bar{v}_{i}, \bar{v}_{j}\right)=\varangle\left(v_{i}, v_{j}\right)$ for all $i, j$. From the description of $(n+1)$-packers of $D_{k}^{n}(\pi)$ we conclude that $\operatorname{dist}\left(m, x_{i}\right)=\pi, i=0, \cdots, n$, and that all angles $\varangle\left(v_{i}, v_{j}\right)$ are equal to the spherical distance between the vertices of the maximal regularly inscribed $n$-simplex in $D_{0}^{n}(1)$. In particular, we have pack $_{n+1} S_{m}=$ pack $_{n+1} S_{1}^{n-1}$. This on the other hand, by the characterization of $S_{1}^{n-1}$ given in [GW, Theorem B], implies that $S_{m}$ is isometric to $S_{1}^{n-1}$, i.e., $m$ is a euclidean point of $X$.

We conclude from the above that $\operatorname{seg}(m) \subset D_{k}^{n}(\pi)$, that $\operatorname{dist}\left(x_{i}, x_{j}\right)=$ $\operatorname{dist}\left(\bar{x}_{i}, \bar{x}_{j}\right)$ for all $i, j$, and that $\bar{x}_{0}, \cdots, \bar{x}_{n}$ are the vertices in the maximal regular $n$-simplex $\Delta_{k}^{n}$ inscribed in $D_{k}^{n}(\pi)$. It also follows that the $\bar{x}_{i}$ 's are unique, i.e., there are unique segments $c_{i}=c_{v_{i}}$ from $m$ to $x_{i}, i=0, \cdots, n$. The corresponding segments in $S_{k}^{n}$ will be denoted by $\bar{c}_{i}, c_{i}=\exp _{m} \circ \bar{c}_{i}$. Since $\varangle\left(c_{i}, c_{j}\right)=\varangle\left(\bar{c}_{i}, \bar{c}_{j}\right)$ and $\operatorname{dist}\left(x_{i}, x_{j}\right)=\operatorname{dist}\left(\bar{x}_{i}, \bar{x}_{j}\right)$, it follows by $2.4\left(\mathrm{ii}^{\prime}\right)$ that $c_{i}$ and $c_{j}, i \neq j$, span a triangular surface isometric to the 2-simplex in $D_{k}^{2}(\pi) \subset S_{k}^{n}$ with vertices $m, \bar{x}_{i}$, and $\bar{x}_{j}$, and with totally geodesic interior. In fact, because $\exp _{m}: \operatorname{seg}(m) \rightarrow X$ is distance nonincreasing and 2.4 (i), we may assume that each of these simplices are isometric images under $\exp _{m}$ of the corresponding simplices in $D_{k}^{n}(\pi)$. When $n=2$, the union of the three 2-simplices just described is the desired 2-simplex $\Delta_{k}^{2}$. If $n \geq 2$, consider the configuration of 2 -simplices spanned by $\left(c_{0}, c_{1}, c_{2}\right)$. Fix a point $x$ on the segment joining $x_{0}$ and $x_{1}$ (see Figure 3.4). Distance comparison based on this segment and $x_{2}$ (cf. $\left.2.4\left(\mathrm{i}^{\prime}\right)\right)$ implies that $\operatorname{dist}\left(x_{2}, x\right)$ is not smaller than the corresponding distance in $S_{k}^{2} \subset S_{k}^{n}$. Since $\exp _{m}$ is distance nonincreasing, the opposite inequality holds as well (cf. $\left.2.4\left(i^{\prime}\right)\right)$ : Arguing as before, we see that $\exp _{m}$ provides an isometry between the simplex spanned by $m, \bar{x}$, and $\bar{x}_{2}$ and a simplex spanned by $c_{x}$ and $c_{2}$ with totally geodesic interior in $X$. Varying $x$ along the segment from $x_{0}$ to $x_{1}$ produces an isometric image under $\exp _{m}$ of the 3-simplex in $D_{k}^{n}(\pi)$ with vertices $m, \bar{x}_{0}, \bar{x}_{1}$, and $\bar{x}_{2}$, and with totally geodesic interior. This process continues, until in finitely many steps it is established that $\left(x_{0}, \cdots, x_{n}\right)$ are the vertices of a simplex $\Delta_{k}^{n} \subset X$ which is isometric under $\exp _{m}$ to the simplex $=\Delta_{k}^{n}$ with vertices $\left(\bar{x}_{0}, \cdots, \bar{x}_{n}\right)$ in $D_{k}^{n}(\pi)$. By construction, $m$ is the barycenter of $\Delta_{k}^{n}$ and $D(m, \pi)=X$. 


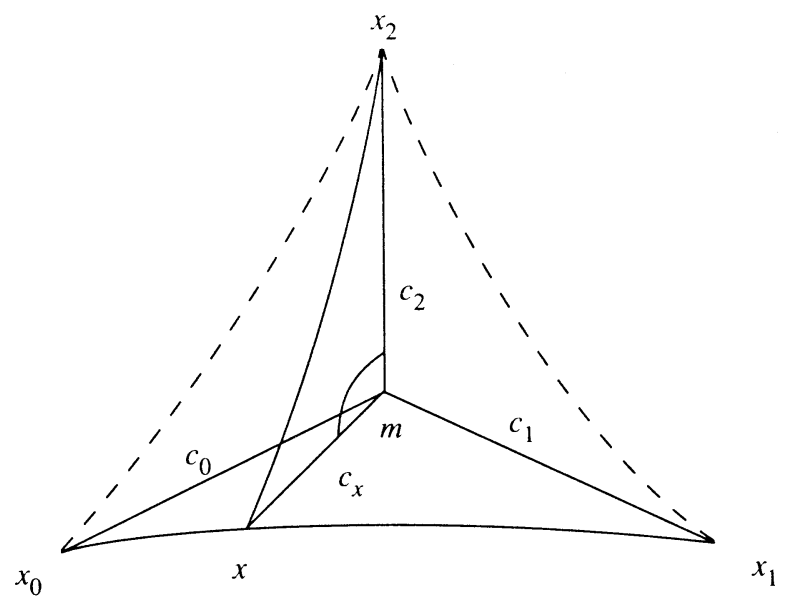

FIGURE 3.4

In general the conclusion of Lemma 3.3 is the best we can hope for. Indeed $X=\Delta_{k}^{n}$ is an $n$-dimensional Alexandrov space with $\operatorname{curv} X \geq k, k \leq$ $\frac{1}{4}, \operatorname{rad} X=\pi$, and $\operatorname{pack}_{n+1} X=\operatorname{pack}_{n+1} D_{k}^{n}(\pi)$. Note also that for $q \leq n$ and pack $_{q+1} X=\operatorname{pack}_{q+1} D_{k}^{n}(\pi)$ one concludes (with the same proof, cf. [GW]) that $X$ contains a $q$-simplex $\Delta_{k}^{q}$.

If in addition $X$ is a closed manifold (or a Poincaré duality space), however, we have the following crucial fact.

Lemma 3.5. Let $X$ be an Alexandrov space as in 3.3 and assume in addition that $X$ is a closed $n$-manifold. Then

(i) there is a point $m_{0} \in X-\Delta_{k}^{n}$ which is equidistant to all vertices of $\Delta_{k}^{n}$. Moreover

(ii) the distance from the barycenter $m$ in $\Delta_{k}^{n}$ to $\partial \Delta_{k}^{n}$ is $\leq \frac{\pi}{2}$.

Proof. Let $x_{0}, \cdots, x_{n} \in \Delta_{k}^{n} \subset X$ be the vertices of $\Delta_{k}^{n}$ and consider the map $F: X \rightarrow \mathbb{R}^{n+1}$ defined by

$$
F(x)=\left(\sum_{i=0}^{n} \operatorname{dist}\left(x_{i}, x\right)\right)^{-1}\left(\operatorname{dist}\left(x_{0}, x\right), \cdots, \operatorname{dist}\left(x_{n}, x\right)\right)
$$

for all $x \in X$. Clearly $F$ maps into the standard $n$-simplex

$$
\Delta^{n}=\left\{\left(u_{0}, \cdots, u_{n}\right) \mid u_{i} \geq 0, \sum u_{i}=1\right\}
$$

spanned by the canonical basis for $\mathbb{R}^{n+1}$. Since $\Delta_{k}^{n}$ is isometrically embedded by 3.3 , we know the restriction of $F$ to $\Delta_{k}^{n}$ explicitly. In fact, $F: \Delta_{k}^{n} \rightarrow$ $F\left(\Delta_{k}^{n}\right) \subset \Delta^{n}$ is obviously a homeomorphism, and more importantly so is $F$ : $\partial \Delta_{k}^{n} \rightarrow F\left(\partial \Delta_{k}^{n}\right)$. In particular, the restriction of $F$ to $\partial \Delta_{k}^{n} \simeq S^{n-1}$ has degree 1 mod 2. It follows that any $v \in H=\left\{u \in \mathbb{R}^{n+1} \mid \sum u_{i}=1\right\}$ in the bounded component of $H-F\left(\partial \Delta_{k}^{n}\right)$ is in the image $F\left(X-\Delta_{k}^{n}\right)$. Otherwise, $F$ restricted to $X-\operatorname{int} \Delta_{k}^{n}$ would be homotopic to a map $G: X-\operatorname{int} \Delta_{k}^{n} \rightarrow F\left(\partial \Delta_{k}^{n}\right) \simeq S^{n-1}$ 
with $G=F$ on $\partial\left(X-\operatorname{int} \Delta_{k}^{n}\right)=\partial \Delta_{k}^{n} \simeq S^{n-1}$, and therefore $\operatorname{deg}\left(F \mid \partial \Delta_{k}^{n}\right)=0$ in contradiction with the above. This clearly applies to $v=\left(\frac{1}{n+1}, \cdots, \frac{1}{n+1}\right) \in H$ and thus yields a point $m_{0} \in X-\Delta_{k}^{n}$ as claimed in (i).

To prove point (ii) first observe that by (3.1) $\operatorname{dist}\left(m_{0}, x_{i}\right) \geq \operatorname{dist}\left(m, x_{i}\right)=\pi$ for $i=0, \cdots, n$. From this and simple distance comparison in $X$ it also follows that $\operatorname{dist}\left(m_{0}, \partial \Delta_{k}^{n}\right) \geq \operatorname{dist}\left(m, \partial \Delta_{k}^{n}\right)=\operatorname{dist}\left(m, m_{i}\right), i=0, \cdots, n$, where $m_{i}$ is the barycenter of the $i^{t h}$ face of $\Delta_{k}^{n}$. On the other hand, since any $x \in X$ has distance at most $\pi$ from $m$, another distance comparison argument gives that (cf. Figure 3.6)

$$
\max _{x \in X-\Delta_{k}^{n}} \operatorname{dist}\left(x, \partial \Delta_{k}^{n}\right) \leq \max _{\bar{x} \in D_{k}^{n}(\pi)-\Delta_{k}^{n}} \operatorname{dist}\left(\bar{x}, \partial \Delta_{k}^{n}\right)=\pi-\operatorname{dist}\left(m, \partial \Delta_{k}^{n}\right),
$$

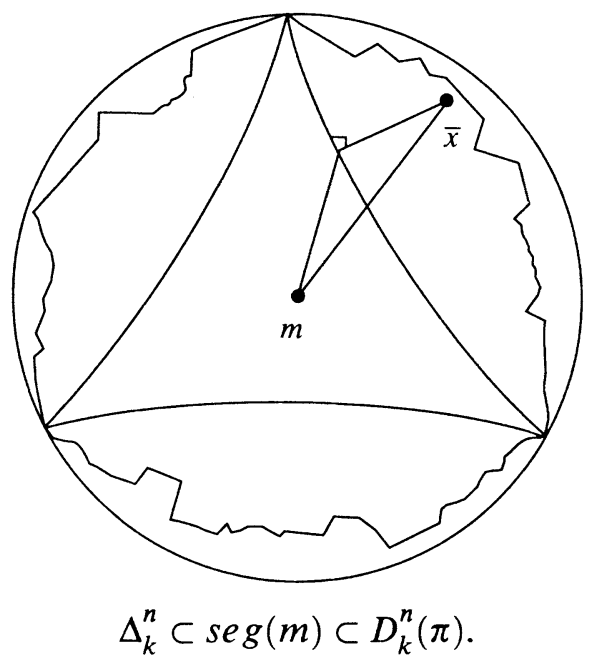

FIGURE 3.6

By combining these inequalities we get especially

$$
\operatorname{dist}\left(m, \partial \Delta_{k}^{n}\right) \leq \pi-\operatorname{dist}\left(m, \partial \Delta_{k}^{n}\right),
$$

i.e., the inradius of $\Delta_{k}^{n}, r_{n}(k)=\operatorname{dist}\left(m, \partial \Delta_{k}^{n}\right) \leq \frac{\pi}{2}$.

As mentioned in the introduction $r_{n}:(-\infty, 1 / 4) \rightarrow(0, \pi)$ is a strictly increasing, continuous, and surjective function. For each $n \geq 2$ let $k_{n} \in$ $(-\infty, 1 / 4)$ be the unique number such that $r_{n}\left(k_{n}\right)=\frac{\pi}{2}$. Note also that the double of $\Delta_{k}^{n}, D \Delta_{k}^{n}$, has radius $\pi$ if and only if $k \leq k_{n}$. In other words pack $_{n+1} X \leq \operatorname{pack}_{n+1} D_{k}^{n}(\pi)$ is optimal also for $n$-dimensional closed Alexandrov manifolds $X$ with curv $X \geq k$ and $\operatorname{rad} X=\pi$ whenever $k \leq k_{n}$. For $\frac{1}{4}>k>k_{n}$, however, there is an $\epsilon=\epsilon(k, n)>0$ such that pack $_{n+1} X \leq$ pack $_{n+1} D_{k}^{n}(\pi)-\epsilon$ for any such $X$. Otherwise, let $\left\{X_{n}\right\}$ be a sequence of closed Alexandrov $n$-manifolds with curv $X_{n} \geq k, \operatorname{rad} X_{n}=\pi$, and pack $_{n+1} X_{n} \rightarrow$ pack $_{n+1} D_{k}^{n}(\pi)$. By the compactness theorem in [BGP] we may assume that $\left\{X_{n}\right\}$ converges to an Alexandrov space $X$ with $\operatorname{curv} X \geq k, \operatorname{rad} X=\pi$, and 
pack $_{n+1} X=\operatorname{pack}_{n+1} D_{k}^{n}(\pi)$. Clearly $\operatorname{dim} X=n$ by 3.3 and hence $X$ must be homeomorphic to $X_{i}, i$ large, according to the stability theorem in [P1]. In particular $X$ is a closed manifold in contradiction with 3.5 since $k>k_{n}$. This proves Theorem D and Corollary E part (2) when $k<\frac{1}{4}$. (Note that $D \Delta_{k}^{n}$ can be approximated by the boundaries of smooth convex "tubular" neighborhoods of $\Delta_{k}^{n}$ in $S_{k}^{n+1}$.)

We now turn to our main result in this section.

Theorem 3.7. Let $X$ be an $n$-dimensional Alexandrov space with curv $X \geq$ $k_{n}, \operatorname{rad} X=\pi$, and pack ${ }_{n+1} X=$ pack $_{n+1} D_{k_{n}}^{n}(\pi)$. If in addition $X$ is a closed manifold (or Poincaré duality space), it is isometric to the double $D \Delta_{k_{n}}^{n}$ of $\Delta_{k_{n}}^{n}$. Proof. Let $m \in \Delta_{k_{n}}^{n}$ and $m_{0} \in X-\Delta_{k_{n}}^{n}$ be as in 3.3 and 3.5. From the proof of 3.5 part (ii) we have

$$
\operatorname{dist}\left(m, \partial \Delta_{k_{n}}^{n}\right) \leq \operatorname{dist}\left(m_{0}, \partial \Delta_{k_{n}}^{n}\right) \leq \pi-\operatorname{dist}\left(m, \partial \Delta_{k_{n}}^{n}\right) .
$$

But from the definition of $k_{n}$ we have $\operatorname{dist}\left(m, \partial \Delta_{k_{n}}^{n}\right)=\pi-\operatorname{dist}\left(m, \partial \Delta_{k_{n}}^{n}\right)$, and hence $\operatorname{dist}\left(m_{0}, \partial \Delta_{k_{n}}^{n}\right)=\operatorname{dist}\left(m, \partial \Delta_{k_{n}}^{n}\right)=\frac{\pi}{2}$. By distance comparison therefore $\operatorname{dist}\left(m_{0}, x_{j}\right) \leq \pi$ for some vertex $x_{j}$. However, $\operatorname{dist}\left(m_{0}, x_{i}\right) \geq$ $\operatorname{dist}\left(m, x_{i}\right)=\pi$ for all $i$ as observed in the proof of 3.5 (ii). Thus $\operatorname{dist}\left(m_{0}, x_{0}\right)$ $=\cdots=\operatorname{dist}\left(m_{0}, x_{n}\right)=\pi$ by $3.5(\mathrm{i})$. We can now repeat the argument in 3.3 whereby deducing that $X$ contains another copy of $\Delta_{k_{n}}^{n}$, with the same vertices but with center $m_{0}$. Moreover, using $D(m, \pi)=X$ it is easy to see that $m$ and $m_{0}$ are joined by $(n+1)$ segments of length $\pi$, the first, resp. last, half of which are contained in the " $m$ "-simplex, resp." $m_{0}$ "-simplex. Consequently, $X=D \Delta_{k_{n}}^{n}$ as claimed.

This result combined with the stability theorems in [GPW], $n \neq 3$, or [P] completes the proof of part (3) and all of Corollary $E$ in the introduction when $k<\frac{1}{4}$.

To complete the proof of Theorem D and Corollary E we now consider $n$ dimensional Alexandrov spaces $X$ with $\operatorname{curv} X \geq k, k \in\left[\frac{1}{4}, 1\right]$, and $\operatorname{rad} X=$ $\pi$.

Now for $k \geq \frac{1}{4}, \operatorname{pack}_{n+1} D_{k}^{n}(\pi)=\operatorname{pack}_{n+1} D_{k}^{n}(\pi / 2 \sqrt{k})=\operatorname{pack}_{n+1} S_{k}^{n-1}$ is realized by the vertices of the maximal regular euclidean $n$-simplex inscribed in $D_{0}^{n}(1 / \sqrt{k})$ (cf. [GW, 2.4]). By Theorem B in [GW], any $n$-dimensional Alexandrov space $X$ with curv $X \geq k \geq \frac{1}{4}$ and pack $_{n+1} X=\operatorname{pack}_{n+1} S_{k}^{n-1}$ is isometric to either $D_{k}^{n}(\pi / 2 \sqrt{k})$ or to $S_{k}^{n}$, neither one of which is a manifold with diameter $\pi$, except for $S_{1}^{n}$. This proves Theorem D and Corollary E when $k \geq \frac{1}{4}$.

Remark 3.8. The arguments in this section apply as well to $n$-dimensional Alexandrov spaces $X$ with $\operatorname{curv} X \geq k, \operatorname{rad} X=\pi$, and $x t_{n+1} X=x t_{n+1} D_{k}^{n}(\pi)$ as long as $x t_{n+1} D_{k}^{n}(\pi)$ is realized by the vertices of $\Delta_{k}^{n} \subset D_{k}^{n}(\pi)$. Although this is the case for $k \leq \frac{1}{16}$ we note, however, that already $k_{3}=\frac{1}{9}$. 


\section{APPENDIX. RIGIDITY FROM DISTANCE COMPARISON in AleXandrov spaces}

The purpose here is to prove the rigidity versions (ii) and (ii ${ }^{\prime}$ ) of the global distance comparison Theorem 2.4. The hinge version (ii') is a well-known and powerful tool in riemannian geometry, where its proof is based on the proof of the corresponding version (ii) (cf., e.g., [CE] or [G]). Our proof here follows directly from $\left(i^{\prime}\right)$, which, on the other hand, only uses the statement (i) (for $X$ and its spaces of directions), not its proof.

Proof of $2.4\left(\mathrm{i}^{\prime}\right)$. Given a triple $\left(x_{0}, x_{1}, x_{2}\right)$ in $X$ and a segment $c_{0}$ from $x_{1}$ to $x_{2}$ as in $2.4(\mathrm{i})$, assume as in $2.4\left(\mathrm{i}^{\prime}\right)$ that

$$
\operatorname{dist}\left(x_{0}, c_{0}\left(t_{0}\right)\right)=\operatorname{dist}\left(\bar{x}_{0}, \bar{c}_{0}\left(t_{0}\right)\right)
$$

for some $t_{0} \in\left(0, \operatorname{dist}\left(x_{1}, x_{2}\right)\right)$, and let $c_{t_{0}}$ be a segment from $x_{0}$ to $c_{0}\left(t_{0}\right)$ in $X$. Fix an arbitrary $s \in\left(0, \operatorname{dist}\left(x_{0}, c_{0}\left(t_{0}\right)\right)\right)$ and consider segments from $c_{t_{0}}(s)$ to $x_{1}$ and to $x_{2}$ (as in Figure A.1).

Observe first that the angles at $c_{0}\left(t_{0}\right)$ are equal to their comparison angles, because they cannot be smaller (2.4(ii)), and their sum is $\pi$. As a consequence (2.4 (i),(ii)), all segments from $c_{t_{0}}(s)$ to $x_{1}$ and $x_{2}$ have lengths as in the comparison space $S_{k}^{2}$. Applying the argument for the angles at $c_{0}\left(t_{0}\right)$ from before to $c_{t_{0}}(s)$, we see that all angles at $c_{t_{0}}(s)$ involving $x_{1}$ (resp. $\left.x_{2}\right)$ are as in the comparison space. A combination of standard angle comparison and the triangle inequality then shows that the angle of $c_{t_{0}}(s)$ between the segments to $x_{1}$ and $x_{2}$ is also as in the comparison space, i.e., it is the sum of two hinge angles. This proves that the segments to $x_{1}$ and $x_{2}$ are unique: Indeed, the existence of more than one segment to one of the points $x_{i}$ would cause bifurcation of geodesics in the space of directions $S_{c_{t_{0}}(s)}$. This, however, is impossible since $S_{c_{t_{0}}(s)}$ is itself an Alexandrov space.

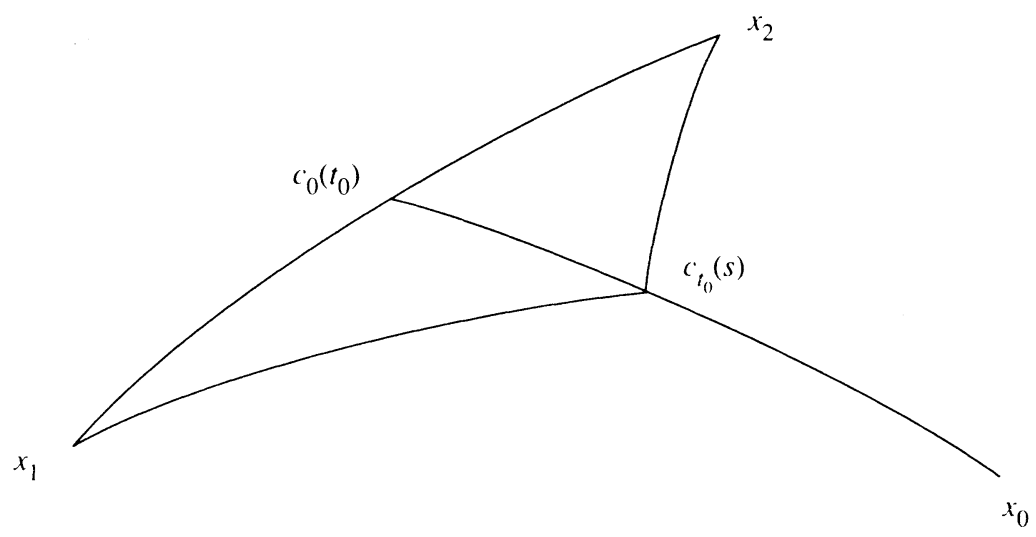

Figure A.1 


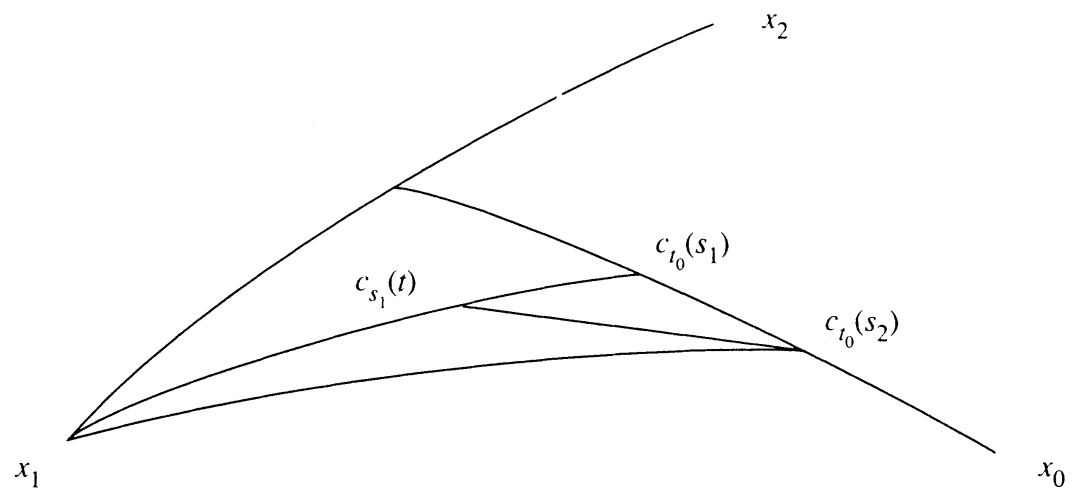

Figure A.2

We now claim that the unique family of segments to $x_{1}$ and $x_{2}$ along the interior points of $c_{t_{0}}$, together with their limit segments at $x_{0}$, constitute the desired surface.

First consider the part swept out from $x_{1}$. We claim that the angles at $x_{1}$ between any two segments to $c_{t_{0}}$ is as in the comparison triangle (see Figure A.2).

Pick any interior point $c_{s_{1}}(t)$ on the segment from $x_{1}$ to $c_{t_{0}}\left(s_{1}\right)$ and join it to $c_{t_{0}}\left(s_{2}\right)$ by a segment (see Figure A.2). By 2.4(i) and (ii) this segment as well has length equal to that of the corresponding segment in $S_{k}^{2}$. Moreover, arguing as before the angles at $c_{s_{1}}(t)$ are the same as in the model. A simple limit argument then shows that the angle at $x_{1}$ between the unique segments from $x_{1}$ to $c_{t_{0}}\left(s_{1}\right)$ and to $c_{t_{0}}\left(s_{2}\right)$ is as in the model surface. It is now evident from 2.4 (i) and (ii) that this half of the surface is isometrically embedded in $X$. Moreover, since geodesics cannot bifurcate in $X$ we see that the interior of this triangular surface is totally geodesic, in fact any two points of it are joined by a unique segment in $X$ and this segment lies in the surface. For the same reasons we know that the other half of the surface, i.e., the one containing $x_{2}$, is isometrically embedded in $X$ with totally geodesic interior.

Finally, we need to see that the two triangular surfaces together form the desired surface. Fix two interior points $c_{0}\left(t_{1}\right)$ and $c_{0}\left(t_{2}\right)$ on either half of $c_{0}$, and join them to $x_{0}$ by segments in the surfaces just constructed (see Figure A.3). By the triangle inequality, the angle between these segments does not exceed the corresponding angle in the model surface. However, by angle comparison, it cannot be smaller. It is now clear, again using 2.4(i) and (ii), that the distance between any pair of points on the segments considered is as in the model. Moreover, between any two interior points of the union of the two triangular surfaces there is a unique segment in $X$, and this segment lies in the union of these surfaces. This completes the proof.

We now proceed to consider the hinge version of 2.4. 


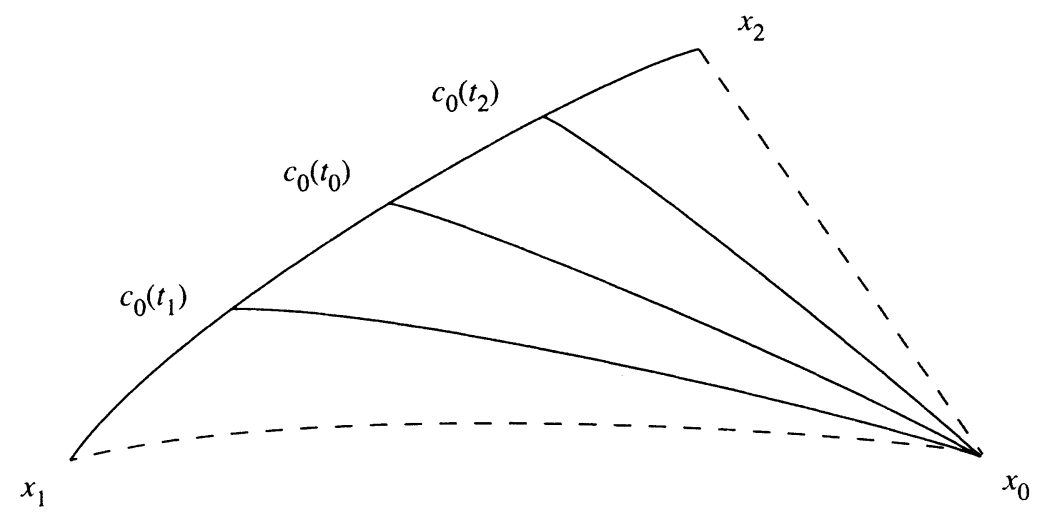

Figure A.3

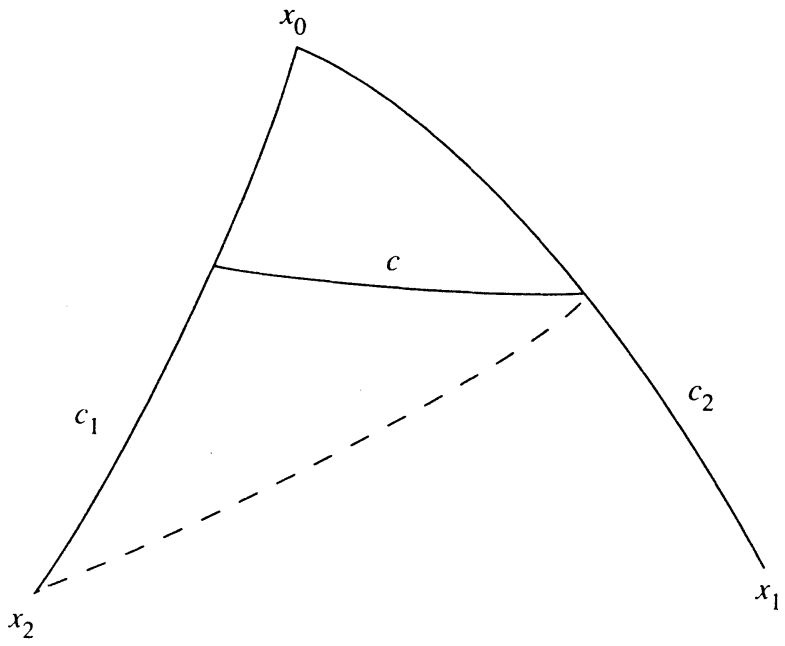

Figure A.4

Proof of $2.4\left(\right.$ ii $\left.^{\prime}\right)$. Given a hinge $\left(x_{0}, c_{1}, c_{2}\right)$ and a segment $c$ between an interior point of $c_{1}$ and an interior point of $c_{2}$ (see Figure A.4), then, by assumption and 2.4 (i) and $\left(i^{\prime}\right)$, the length of $c$ is equal to the length of the corresponding segment in the comparison hinge in $S_{k}^{2}$. Observe that for the same reason the distance from $x_{2}$ to the endpoint of $c$ on $c_{2}$ equals the corresponding distance in $S_{k}^{2}$.

Now consider the surface generated by $c$ and $c_{1}$ as in $2.4\left(i^{\prime}\right)$. Let $\tilde{c}$ be the edge in this triangular surface opposite $x_{0}$. Applying $2.4\left(i^{\prime}\right)$ to $\tilde{c}$ and $c_{2}$ yields the desired surface. It is easy to check that the half of this triangular surface which contains $x_{0}$ coincides with the surface generated by $c$ and $c_{1}$. This, therefore, completes the proof. 


\section{REFERENCES}

[AG] U. Abresch and D. Gromoll, On complete manifolds with non-negative Ricci curvature, J. Amer. Math. Soc. 3 (1990), 355-374.

[Be] V. N. Berestowski, Spaces with bounded curvature and distance geometry, Sibirsk. Mat. Zh. 27 (1986), 26-34.

[BGP] Y. Burago, M. Gromov, and G. Perelman, A.D. Alexandrov's spaces with curvatures bounded from below I, Uspekhi Mat. Nauk 47 (1992), 3-51; English transl., Russian Math. Surveys 47 (1992), 1-58.

[CE] J. Cheeger and D. Ebin, Comparison theorems in Riemannian geometry, North-Holland Math. Library, vol. 9, North-Holland, Amsterdam and New York, 1975.

[C] H. S. M. Coxeter, Introduction to geometry, Wiley, New York, 1961.

[F] I. Fary, Sur la courbure totale d'une courbe gauche faisant un naeud, Bull. Soc. Math. France 77 (1949), 128-138.

[GG] D. Gromoll and K. Grove, A generalization of Berger's rigidity theorem for positively curved manifolds, Ann. Sci. École. Norm. Sup. 20 (1987), 277-239.

[G1] M. Gromov, Groups of polynomial growth and expanding maps, Inst. Hautes Études Sci. Publ. Math. 53 (1981), 53-73.

[G2] - Filling Riemannian manifolds, J. Differential Geom. 18 (1983), 1-148.

[G] K. Grove, Metric and topological measurements of manifolds, Proc. Internat. Congr. Math. (Kyoto, Japan, 1990), Math. Soc. Japan and Springer-Verlag, Berlin and New York, 1991, pp. 511-519.

[GM] K. Grove and S. Markvorsen, Curvature, triameter and beyond, Bull. Amer. Math. Soc. (N. S.) 27 (1992), 261-265.

[GP1] K. Grove and P. Petersen V, Bounding homotopy types by geometry, Ann. of Math. (2) 128 (1988), 195-206.

[GP2] _ Homotopy types of positively curved manifolds with large volume, Amer. J. Math 110 (1988), 1183-1188.

[GP3] __, Manifolds near the boundary of existence, J. Differential Geom. 33 (1991), 379-394.

[GP4] _ A pinching theorem for homotopy spheres, J. Amer. Math. Soc. 3 (1990), 671-677; On the excess of metric spaces and manifolds, preprint UMCP, 1989.

[GP5] _- Volume comparison à la Alexandrov, Acta Math. 169 (1992), 131-151.

[GP6] K. Grove and P. Petersen, A radius sphere theorem, Invent. Math. 112 (1993), 577-583.

[GPW] K. Grove, P. Petersen V, and J.-Y. Wu, Geometric finiteness theorems via controlled topology, Invent. Math. 99 (1990), 205-213; Erratum, Invent. Math. 104 (1991), 221-222.

[GW] K. Grove and F. Wilhelm, Hard and soft packing radius theorems, preprint.

[H] R. S. Hamilton, Three-manifolds with positive Ricci curvature, J. Differential Geom. 17 (1982), 255-306.

[HK] W.-Y. Hsiang and B. Kleiner, On the topology of positively curved 4-manifolds with symmetry, J. Differential Geom. 29 (1989), 615-621.

[N] F. Nielsen, On the sum of distances between n points on a sphere, Nordisk Mat. Tidsskr. 13 (1965), 45-50. (Danish)

[OSY] Y. Otsu, K. Shiohama, and T. Yamaguchi, A new version of differentiable sphere theorem, Invent. Math. 98 (1989), 219-228.

[P] G. Perelman, Alexandrov's spaces with curvatures bounded from below II, preprint.

[Pt1] P. Petersen V, A finiteness theorem for metric spaces, J. Differential Geom. 31 (1990), 387-395.

[Pt2] _ Gromov-Hausdorff convergence of metric spaces, Proc. Sympos. Pure Math., vol. 54, Part 3, Amer. Math. Soc., Providence, RI, 1993, pp. 489-504.

[PI] C. Plaut, Spaces of Wald curvature bounded below, J. Geom. Anal. (to appear). 
[R] W. Rinow, Die Innere Geometrie der Metrischen Räume, Springer-Verlag, Berlin and New York, 1961.

[Y] T. Yamaguchi, Collapsing and pinching under a lower curvature bound, Ann. of Math. (2) 133 (1991), 317-357.

Department of Mathematics, University of Maryland, College Park, Maryland 20742

E-mail address: kng@math.umd.edu

Technical University of Denmark, Lyngby, Denmark

E-mail address: steen@mat.dtu.dk 